\title{
Electron emission contributions to dark current and its relation to microscopic field enhancement and heating in accelerator structures
}

\author{
Kevin L. Jensen \\ Naval Research Laboratory, Washington, D.C. 20375-5347, USA \\ Y. Y. Lau \\ University of Michigan, Ann Arbor, Michigan 48109, USA \\ D. W. Feldman and P. G. O'Shea \\ University of Maryland, College Park, Maryland 20742, USA
}

(Received 25 February 2008; published 26 August 2008)

\begin{abstract}
Analytically tractable models of thermal-field emission, field enhancement, and heating mechanisms (Nottingham and resistive) are developed and combined to make estimates of the fields and temperatures that accompany the development and growth of asperities. The relation of asperity dimensions to dark current is discussed in two experimentally motivated examples. The hypothetical relation of microscopic sources of dark current and heating to breakdown is discussed in the context of Nottingham and resistive heating. The latter are estimated using a general thermal-field methodology. A point-charge model is used to find field enhancement factors. Last, a thermal model is used to estimate the temperature dependence of the resistivity and thermal conductivity. Together, these models suggest that conditions can arise in which the temperature at the apex of an asperity can experience growth and contribute to melting or fracture (or both), and that Nottingham heating generally dominates the resistive heating term.
\end{abstract}

DOI: 10.1103/PhysRevSTAB.11.081001

PACS numbers: 79.70.+q, 52.80.Vp, 52.59.-f, 79.40.+z

\section{INTRODUCTION}

Dark current, or the unwanted emission of electrons from the surface of both photocathodes and metal surfaces within an rf photoinjector as a consequence of thermalfield emission, has long been observed (generally, with dismay) in the development of free-electron lasers [1,2] and accelerators. It is undesirable, not only because it limits operational performance [3-10], but because it also plays a role in breakdown [11-18]. Though much is known about the relationship between dark current (thermal-field emission), local heating of asperities, field enhancement, and their failure mechanisms, good theoretical models are hampered by a lack of emission models that are correct in the parameter regime of both high fields and temperatures, flexible field enhancement models, and the relationship of temperature and field enhancement to resistive (i.e., heating due to electron-phonon scattering) and Nottingham (i.e., excess energy given up by an electron at the Fermi level scattering to occupy a state below the Fermi level vacated by a field emitted electron) heating for conditions that occur.

The examination of the origin of dark current is often in the context of the Fowler-Nordheim (FN) equation [19] (see the Appendices) coupled with models of field enhancement that are numerically inferred. Such approaches are appropriate to the phases of dark current modeling $[9,15,16,20,21])$ prior to large temperature excursions, but their applicability afterwards is undermined by (i) when temperatures at the emission site approach the melting point of the metals (emission is thermal field rather than FN); (ii) when migration of material results in nanoprotrusions that dynamically grow but whose size precludes their adequate consideration by numerical means; and (iii) when the only acknowledgment of geometry is through the positing of a field enhancement "beta factor" without considering its companion effect on the "notional" emission area [22]. That such considerations matter is supported by treatments in which the thermal-field emission for multidimensional structures is numerically found $[23,24]$ and which capture effects absent in the analytical approaches.

The intent of the present work is to develop a methodology that can provide estimates of the current levels involved at the asperities and the associated heating that occurs as a consequence, but that is respectful both of the emission current physics and the evolving geometry. A reliance on flat (that is, one-dimensional) and conventional field and thermal emission models and their neglect of area and field enhancement and area factors associated with electron emission in treatments of dark current are incomplete and occasionally incorrect. The methodologies that are brought to bear to rectify this have been vetted in the modeling and characterization of modern electron sources. A correct description entails several requirements: (i) when heating is significant, models transcending the Fowler- 
Nordheim equation are needed; (ii) a model of field enhancement that evolves as conditions evolve is required; and (iii) models describing "run-away" mechanisms leading to failure calculate resistive and Nottingham heating contributions correctly. To meet these demands, a framework that couples the disparate phenomena and which favors analytical rather than numerical methods to address thermal-field emission from evolving protrusions subject to heating mechanisms related to electron emission, in particular, is developed.

\section{LOCALIZED HEATING DUE TO EMISSION AND ITS RELATION TO BREAKDOWN: AN HYPOTHESIS}

Heat localization is associated with the appearance of nanoprotrusions and sharp ridges on field emitter tips [18], and the development of such structures are considered by the field emission community to be a precursor to breakdown in field emitter arrays in that asperity creation is in part due to ion bombardment of the emitter surface [25]. Surface migration of atoms or clusters of atoms ("protrusions" in the parlance of Charbonnier) becomes possible when temperature and field conditions favor it: considerable literature has been devoted to the theoretical study and experimental characterization of it and its relation to electron emission [26]. Ancona [27] in particular argues, in his study of metal field emitter failure, that his findings suggest melting accompanies tip failure rather than triggers it. Temperature increases can be the consequence of the impact of energetic ions, and Nottingham and resistive heating mechanisms, the exact nature of which shall be explored in detail in subsequent sections. Analogously in the accelerator community, Wilson has hypothesized that surface melting accompanies breakdown [28-30], and Norem et al. $[15,16]$ observe that fracture can occur at the same sharp regions that lead to field emission and local heating. Insepov et al. [31] have performed molecular dynamic simulations that produce behavior supportive of the surface migration hypothesis. While the accelerator community has not converged upon an accepted underlying microscopic mechanism for local heating and melting, or its relation to origins of mechanical fatigue, and the sequence of events leading to breakdown [6], the models advocated by Wilson, Norem, Bonin, and others contain explicitly and implicitly speculations regarding the impact of field emission and temperature-induced surface migration (though other mechanisms, such as back-streaming ions [16], contribute).

In considering the current flow heating hypotheses, an account of heating mechanisms merits revisiting. The present analysis seeks to quantify otherwise qualitative speculations that deal with electron emission at localized, likely nanoscale, emission sites thought to accompany breakdown. Consequently, conditions existing in the microwave cavities (e.g., background pressure, microwave power, device dimensions, and so on $[32,33]$ ) are of secondary importance to the methods developed herein in spite of their generally paramount importance in the literature. Field emission electrons tunnel through surface barriers on femtosecond time scales, and the time duration between individual electron emission events (each of which contributes to Nottingham heating and cooling) from a $10 \mathrm{~nm}^{2}$ area for current densities on the order of $10^{8} \mathrm{~A} / \mathrm{cm}^{2}$ is $16 \mathrm{fs}$ on average. Compare these "nanophysics" time scales to others that matter in the evolution of dark current. First, heat diffusion in metals (e.g., copper) is characterized by a heat diffusion time constant $\Delta t=$ $\Delta x^{2} / 4 D_{o}$ on the order of $\mathrm{O}(2 \mathrm{~ns})$ for a $\mathrm{O}(1 \mu \mathrm{m})$ migration scale distance. Second, the time scale of the variation in the magnitude of the electric field at the surface of a conductor is related to the inverse frequency and therefore on the order of $0.5 \mathrm{~ns}$ in an S-band photoinjector or on the order of $1.4 \mathrm{~ns}$ in photoinjectors for high power free-electron lasers under development [34].

Thus, Nottingham heating and field emission phenomena (see Sec. VI) involve time scales so rapid that little is compromised by assuming "static" conditions in the emission and heating equations to be developed (a point made by Bonin [35]). Such would not be the case if the focus were on surface melting and cone formation (precursors to breakdown), as in other studies [36-38], but it is so if the focus is on the applicability of the emission equations and their relationship to Nottingham heating at the emission sites plus methods to estimate field enhancement effects from structures whose relevant dimensions can be orders of magnitude removed from the dimensions of melted regions that follow in the wake of a breakdown event. Insofar as repeated heating and cooling may be a component of mechanical fatigue that leads to rf breakdown, then other correlations, such as with rf frequency, may exist with breakdown, but in the context of electron emission from nanoscale features, such correlations are not addressed in the present analysis.

\section{CHARACTERIZATION OF DARK CURRENT DATA: TWO EXAMPLES}

The context of methods developed within the present work to characterize and assess dark current are illustrated by two examples, presented before the methodology so as to indicate and justify its applicability. The first considers dark current measured under high gradient testing of high frequency structures for accelerators, The second considers dark current observed on a $\mathrm{CsK}_{2} \mathrm{Sb}$ photocathode used in a free-electron laser. The comparison is based on representing the emitter surface using a point-charge model in which a linear chain of point charges is assembled so that their equipotential surface mimics that of an actual emitter, as described in Sec. V. By themselves the radius of the equipotential line of the $n$th point charge, dubbed $a_{n}$, shall be related to the magnitude of the charge itself. 


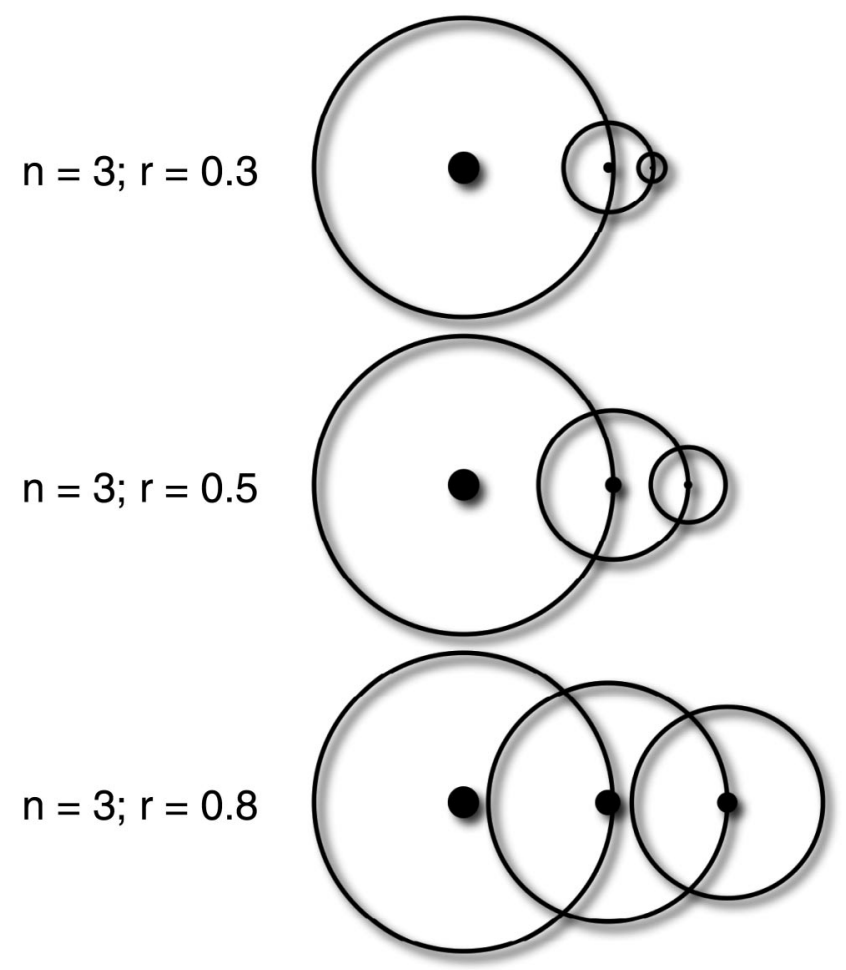

FIG. 1. The creation of an emitter in the point charge model for $n=3$ and various values of $r$. The circles are the equipotential lines of their center charge only: the total equipotential surface is created by all the point charges and is therefore slightly larger.

Assembling these charges in a line approximates the emitter, as in Fig. 1 in the case of 3 point charges $(n=3)$, such that the $n$th point charge sits on the apex of the $(n-1)$ th sphere. The field and apex radius can be ascertained analytically if $a_{n+1} / a_{n}=r$ for all $n$, and such an example is

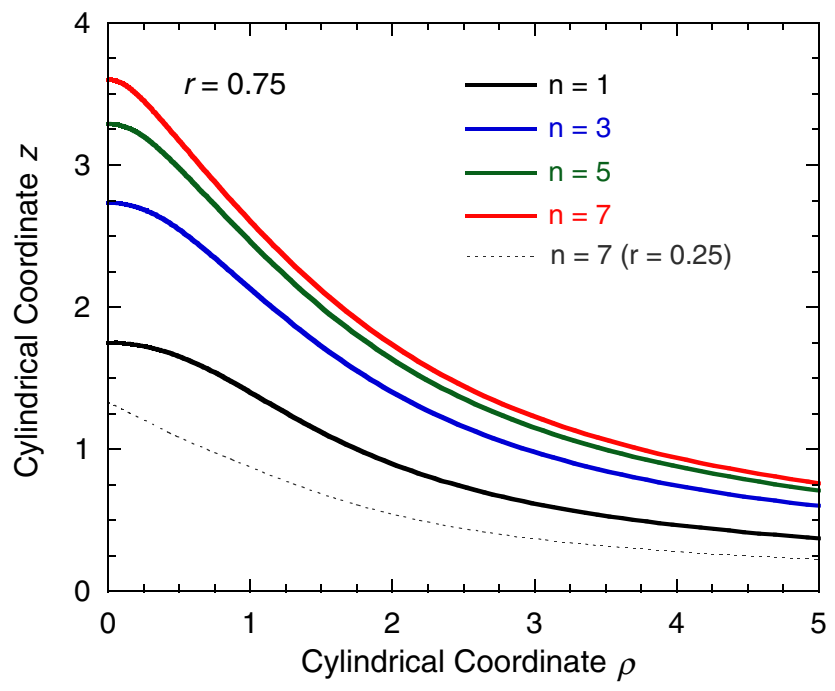

FIG. 2. (Color) The shape of the emitter in the point-charge model for different values of $n$ for $r=0.75$. Also shown is the $n=7, r=0.25$ surface. shown in Fig. 2 for $r=0.75$ for various $n$. The growth as well as the destruction of metallic whiskers or bumps can therefore be characterized by evolution of $n$ and $r$. If the apex of an emitter sharpens due to surface migration, such as occurs under high fields and accelerated when the emitter is hotter, then the $r$ factor should decrease. Conversely, if the apex is damaged and blunted, then the $n$ factor should decrease. Insofar as the field enhancement factor evolves-as was explicitly shown for blunting in a back ion bombardment environment that degrades field emitters $[39,40]$ - such a characterization has use in the examination of the evolution of dark current (the related evolution of the statistics of field enhancement variation, discussed in the cited references, is too far afield in the present work, so the assumption that all contributing nanostructures have identical geometric features is implicitly made here).

In the first example, Wang et al. [41], in tests at SLAC on an X-band accelerating section built at CERN, monitored dark current during high gradient conditioning experiments. In Fig. 2 of that reference, two data sets (120 MV/m "upstream" and "downstream") are reproduced in Fig. 3 and compared to the model developed in Sec. V using, in particular, Eq. (9). Wang et al. considered the average accelerating gradient in the representation of their data; the peak (or actual macroscopic) gradient at the surface was larger, such that an average field of $120 \mathrm{MV} / \mathrm{m}$ corresponded to a peak field of $285 \mathrm{MV} / \mathrm{m}$ (recall that for field emission, the peak value matters). A qualitative difference in the trends associated with the "up" (open circle) and "down" (closed circle) data sets in terms of shape and magnitude. At the high field limit, both curves drop away from the exponential trend of the

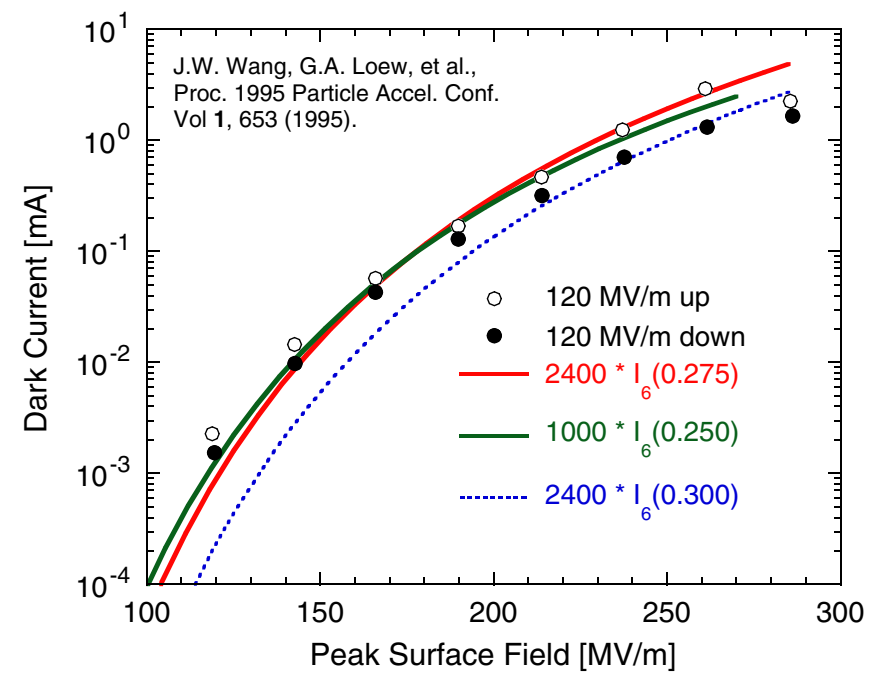

FIG. 3. (Color) Characterization of the dark current measured by Wang and Loew in Ref. [41] with the point-charge model. The red, blue, and green theory lines are identified by the nomenclature $N I_{n}(r)$, where $N$ acts as the number of emitters, $I$ indicates current, and $r$ is the ratio factor. 
data. It was found that the " $120 \mathrm{MV} / \mathrm{m}$ upstream" data was well modeled by assuming $N=2400$ whiskers characterized by a base radius of 10 microns (i.e., $a_{0}=$ $10 \mu \mathrm{m}), n=6$, and $r=0.275$, and otherwise using copper material parameters (e.g., work function of $4.5 \mathrm{eV}$ ), as shown by the red line. The trend of the downstream whiskers is matched by $N=1000$ emitters whiskers characterized by $n=6$, and $r=0.250$, as shown by the blue line. As with field emitter arrays and nanotubes, distributions of emitters exist with the sharpest emitters providing the majority of the current. Methods known to eliminate nanoprotrusions and result in more uniform but less sharp tips in arrays, due to surface migration or sputtering (see Sec. II.F.4 of Ref. [44]), include the conditioning of Spindttype emitters or operating them in a glow discharge $[42,43]$. It may be naively expected that the trends of $N$ and $r$ indicate likewise here, but that would be in error: it is open speculation as to the processes responsible for differences in $r$ and $N$, though tellingly the $r$ values are not significantly different, and $N$ is expected to vary because some current is invariably lost as the beam passes through accelerator structures. Further, other processes affect the magnitude of the dark current, such as operational frequency (though for recent studies in the tens of $\mathrm{GHz}$, the maximum obtainable surface field was found to be independent of temperature or frequency [45]).

In the second example, in 1991, the Los Alamos freeelectron laser (FEL) was rebuilt with a photoelectric injector to enable a lower emittance and brighter beam. Measurements of the high-brightness accelerator FEL (HIBAF) system were conducted to assess temporal and spatial beam characteristics for $10 \mathrm{nC}$ per micropulse for both single micropulses and macropulses. In the course of these studies, dark current from the photocathode was found to occur, and argued to be caused by field emission $[1,2]$ from the $\mathrm{CsK}_{2} \mathrm{Sb}$ photocathode itself. In contrast to field emission from metallic whiskers, photoemission and field emission from semiconductors is more difficult to model, due to a combination of factors, such as electron affinity, band gap, the existence of band bending, and greatly reduced carrier concentrations in the conduction band of the semiconductor or emission from the valence band and surface states [46,47]. The methodology of a recently developed model of photoemission from $p$-type semiconductors [48] was adapted for use here, possible because the calculation of quantum efficiency (QE) for a photocathode makes use of emission physics that bears directly on the field emission problem itself. The emission barrier for $p$-type semiconductors is the sum of the band gap and the electron affinity. The Schottky barrier lowering (the "image charge") is not included because of the behavior of the transmission probability for low triangular barriers and the reduction in the Schottky factor due to the dielectric constant: these and other factors particular to semiconductors (e.g., oxides and surface states) cause ambiguity in the barrier magnitude, and it is widely appre- ciated that an overestimated barrier results in an underestimated apex radius. The focus on geometry and field enhancement commends the simpler model.

The multi-alkali materials catalogued by Sommer and Spicer [49] suggests a value of $1.1 \mathrm{eV}$ for the band gap and constrain the electron affinity to larger than $0.6 \mathrm{eV}$. The photoemission data of Michelato et al. [50] and the measured QE of 4\% of the LANL photocathode (which was as high as $8 \%$ [1]) at $537 \mathrm{~nm}$ drive laser wavelength is also shown. A recently developed theoretical model of quantum efficiency developed for the analysis of $\mathrm{Cs}_{3} \mathrm{Sb}$ [48] was used with modifications reflecting band gap, and potassium mass, but otherwise adopting the same parameters, to conclude that the electron affinity is $0.65 \mathrm{eV}$ based on the experimental data, as shown in Fig. 4. We note that differences in overall scale factor are to be expected, given the reliance on the dielectric parameters used from the $\mathrm{Cs}_{3} \mathrm{Sb}$ simulations, which are analogous to but not identical with $\mathrm{CsK}_{2} \mathrm{Sb}$ : it is seen that, apart from the factor of 2 difference in scale, the theory and experimental data are in good agreement for the electron affinity chosen. With the semiconductor values in hand, and using the triangular barrier semiconductor field emission model (heating is not expected to be significant due to repetition rate and pulse duration, so a general thermal-field equation would be excessive) in the dark current simulation codes, the experimental field emission observed in the HIBAF accelerator was analyzed, and is shown in Figs. 5 and 6. The unconventional dependence of the $y$-axis coordinate to the third power of the macroscopic field $F_{o}$ is a consequence of the dependence on the notional emission area's field dependence, as discussed in the Sec. V. The parameters so obtained indicate that the agreement is good and supports

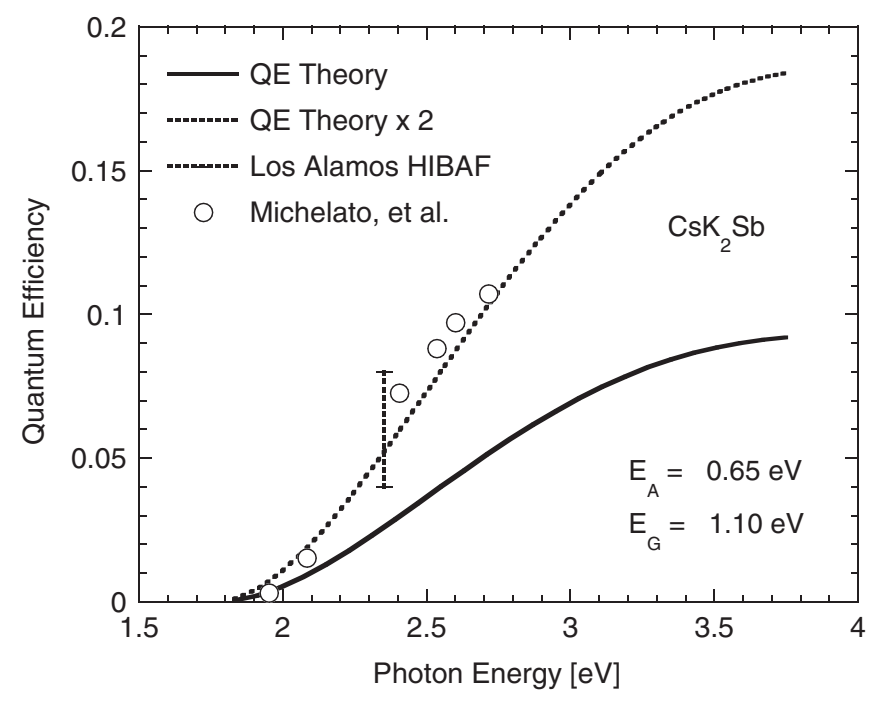

FIG. 4. Comparison of experimentally measured values of the quantum efficiency of $\mathrm{CsK}_{2} \mathrm{Sb}$ photocathodes based on Refs. [50] (circles) and [1] (dashed line) compared to the theoretical model, from which an operational value for the electron affinity is deduced to be $0.65 \mathrm{eV}$. 


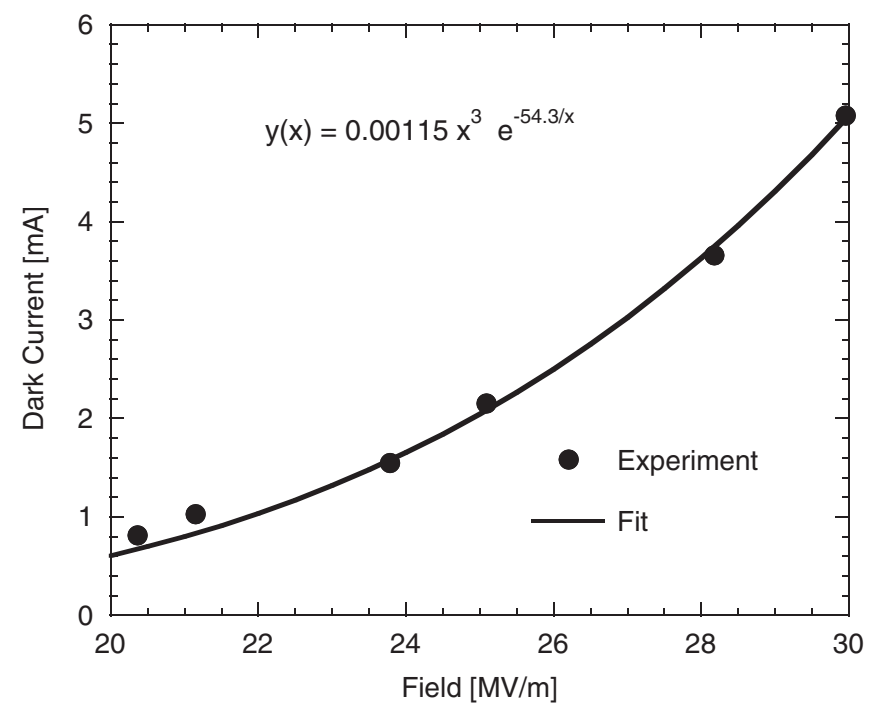

FIG. 5. Dark current in milliamps measured from the $\mathrm{CsK}_{2} \mathrm{Sb}$ photocathode surface in the Los Alamos high-brightness accelerator FEL (HIBAF). The "fit" is based on the product of a Fowler-Nordheim current density vs field relation with a notional emission area vs field relation (see text).

the attribution of the dark current to field emission. Further empirical evidence that the photocathode dark current was due to field emission was observed when the photocathode surface was polished to an optically flat condition, wherein the total dark current dropped to under a microamp.

The ability to model dark current from both accelerator structures and photocathodes has now been illustrated. We turn to explaining the details of the model and then extending it to examine the question of Nottingham and resistive heating of protrusions due to emitted current.

\section{A GENERAL THERMAL-FIELD EQUATION}

The Fowler-Nordheim (FN) equation (see Appendix A) is inapplicable when temperatures are near the melting point of metals, and evidence of melting and cone formation (dubbed "Taylor cones" by Wilson) on metal surfaces is evidence that such temperatures are experienced. The inadequacy of the FN equation in the thermal-field regime has been discussed by Murphy and Good [51]: for example, using a copperlike $\Phi$ of $4.5 \mathrm{eV}$, and limiting the thermal correction term (in their notation, $c$ is not the speed of light) to satisfy $\pi c k_{B} T \approx\left(2 \pi k_{B} T / \hbar F\right) \sqrt{2 m \Phi} \leq 1$ (terms are defined in Table I) indicates that for a field of $6 \mathrm{GV} / \mathrm{m}$, then $T<1020 \mathrm{~K}$, or $338 \mathrm{~K}$ below the melting point of $\mathrm{Cu}$. For a heated metallic whisker or cusp for which the field drops precipitously away from apex, thermal-field contributions to the emission current are underestimated, thereby underestimating the heating mechanism at the emission sites. A recently developed general thermal-field (GTF) equation can be used over the range of temperatures and fields a metallic asperity should experience. It is [line 2 in Eq. (1) corrects an error in
Refs. [44,52], of a factor of $n^{2}$ attached to $\Sigma(1 / n)$ ]

$$
\begin{gathered}
J_{\mathrm{GTF}}(F, T)=A_{\mathrm{RLD}} T^{2} N\left(\frac{\beta_{T}}{\beta_{F}}, \beta_{F}\left(E_{o}-\mu\right)\right) \\
N(n, s) \approx n^{2} \Sigma\left(\frac{1}{n}\right) e^{-s}+\Sigma(n) e^{-n s},
\end{gathered}
$$

where $n=\beta_{T} / \beta_{F}$ and the methods to evaluate $\beta_{F}$ and $E_{o}$ are described in detail in the references. For pure field emission conditions, $\beta_{F}$ is equivalent to the $c$ and $\beta_{F}\left(E_{o}-\right.$ $\mu)$ to the $b$ of Murphy and Good, but are here evaluated via Eqs. (43) and (47) of Ref. [52]. $J_{\mathrm{GTF}}$ is field dominated when $n>1$ and thermal dominated when $n<1$. A leading order approximation to $\Sigma(x)$ [Eq. (51) of Ref. [52]], though reasonable, lacks the accuracy sought for here and will be reappraised. It can be expressed, however, as

$$
\Sigma(x) \approx \frac{2}{1-x^{2}}+p_{k}\left(x^{2}\right)
$$

where $p_{k}$ is a polynomial of order $k$. Therefore, the general largeness of the companion exponential terms in Eq. (1) when the argument of $\Sigma$ is larger than unity serves to squelch $p_{k}\left(x^{2}\right)$ for $x>1$ : Eq. (1) therefore functions well in the transition region where both thermal and field components are comparable in size. Note that the singularity in $\Sigma(n)$ cancels a similar singularity in $\Sigma(1 / n)$ so that $N(1, s) \approx(s+1) e^{-s}$ and is finite.

The field over a protrusion drops rapidly away from the symmetry axis for conical and ellipsoidal surfaces [53,54]. Consequently, in addition to both thermal and field emission components contributing, the notional emission area [22] changes not only with changing field but also if the apex radius is evolving, as will occur during field forming [55] in which the apex of an emitter blunts or sharpens depending on field as has been observed when emitters are subject to very high emission [56]. It has been a subject of some speculation dating back to Schottky [57], as to how field enhancement factors multiply together when protrusions on top of protrusions can occur: it has been suggested that such "beta factors" are multiplicative [5,21], but as shown by Miller, Lau, and Booske [58] using exact conformal mapping techniques, such is the case only when the second protrusion is small compared to the one upon which it rests. The protrusions that develop spontaneously upon sharpened surfaces can have dimensions not altogether different from the underlying tip [59], whereas the field emission from breakdown sites are speculated to have a nanoprotrusions on microprotrusions feature, alternately called "hypothetical asperities" [21]. If so, an adaptable field enhancement model (preferably analytic) is highly desirable, especially if it allows for describing the growth of a cone as in dynamic breakdown models, analytical estimates of the field enhancement associated with the hypothetical asperities, and an estimate of the area of the nanoscale emission sites. 
TABLE I. Entries are given in the approximate order of their first use. MKSA units are used unless otherwise specified.

\begin{tabular}{|c|c|c|}
\hline Symbol & Meaning & Definition or value [unit] \\
\hline$c$ & Speed of light & $299792458 \mathrm{~m} / \mathrm{s}$ \\
\hline$q$ & Elementary (electron) charge & $1.6021765 \times 10^{-19} \mathrm{C}$ \\
\hline$m$ & Electron mass & $510998.9 \mathrm{eV} / c^{2}$ \\
\hline$k_{B}$ & Boltzmann's constant & $1 /(11604.506 \mathrm{~K} / \mathrm{eV})$ \\
\hline$F$ & Product of elementary charge and electric field & $\mathrm{eV} / \mathrm{nm}$ \\
\hline$T$ & Temperature & $\mathrm{K}$ \\
\hline$\Phi$ & Work function & $\mathrm{eV}$ \\
\hline$A_{\mathrm{RLD}}$ & Richardson (thermionic emission) constant & $120.17349 \mathrm{~A} / \mathrm{K}^{2} \mathrm{~cm}^{2}$ \\
\hline$\beta_{T}$ & Thermal emission energy slope factor & $1 / k_{B} T$ \\
\hline$\beta_{F}$ & Field emission energy slope factor & Eqs. (43) and (47) of Ref. [52] \\
\hline$n$ & In GTF equation, ratio of energy slope factors & $\beta_{T} / \beta_{F}$ \\
\hline$N(n, s)$ & GTF function & Eq. (1) \\
\hline$\Sigma(x)$ & GTF function & Eqs, (2) and (15) \\
\hline$\mu$ & Chemical potential (Fermi level) & $\mathrm{eV}$ \\
\hline$E_{o}$ & Energy parameter in thermal-field emission equation & See Ref. [52] \\
\hline$r$ & $\begin{array}{l}\text { Ratio of radius associated with point charge with next larger radius } \\
\text { (in point-charge model) }\end{array}$ & $a_{n+1} / a_{n}$ \\
\hline$a_{n}$ & Radius associated with $n$th point charge & $\mu \mathrm{m}$ to $\mathrm{nm}$ \\
\hline$z_{n}$ & Sum of $a_{n}$ & Eq. (3) \\
\hline$S_{n}(r)$ & Ratio of $z_{n}$ to $a_{0}$ & Eq. (3) \\
\hline$\lambda_{j}$ & Point-charge magnitude & Eq. (4) \\
\hline$P_{n}(r)$ & Point-charge magnitude and radius parameter & Eq. (5) \\
\hline$Q_{n}(r)$ & Point-charge magnitude and radius parameter & Eq. (6) \\
\hline$\beta_{n}(r)$ & Field enhancement factor & Eq. (7) \\
\hline$a_{\text {tip }}$ & Apex radius & $\mu \mathrm{m}$ to $\mathrm{nm}$ \\
\hline$I_{\text {tip }}$ & Emitted current from microprotrusion & Amp \\
\hline$J_{\mathrm{GTF}}$ & Thermal-field current density & $\mathrm{Amp} / \mathrm{cm}^{2}$ \\
\hline$S_{j}$ & Geometric slope factor in $I_{\text {tip }}$ & Eq. (10) \\
\hline$N(\alpha)$ & Geometric exponential reduction factor & Eq. (10) \\
\hline$\alpha_{j}$ & Factor relating current density on adjacent ribbons & $\alpha_{j} \approx \ln \left(J_{j} / J_{j+1}\right)$ \\
\hline$\Delta E(F, T)$ & $\begin{array}{l}\text { Average } E \text { released by replacement electron occupying state } \\
\text { of one emitted in Nottingham heating }\end{array}$ & Eq. (11) \\
\hline$f_{\mathrm{FD}}(E)$ & Fermi-Dirac distribution function & $\cdots$ \\
\hline$T(E)$ & Quantum transmission (tunneling) probability & $\ldots$ \\
\hline$\Sigma^{\prime}(x)$ & Associated derivative function of $\sum(x)$ & Eq. (17) \\
\hline$N^{\prime}(n, s)$ & Associated derivative function of $N(n, s)$ & Eq. (20) \\
\hline$\Delta \Omega$ & Solid cone angle & Eq. (21) \\
\hline$\kappa(T)$ & Thermal conductivity & $\mathrm{W} / \mathrm{m}-\mathrm{K}$ \\
\hline$r$ & Spherical coordinate (in cone heating model) & $\mathrm{nm}$ \\
\hline$r_{e}$ & Apex radius & $\mathrm{nm}$ \\
\hline$r_{o}$ & Base radius & $\mu \mathrm{m}$ \\
\hline$\rho$ & Resistivity & Ohm-m \\
\hline$T_{o}$ & Temperature of base & $\mathrm{K}$ \\
\hline$W_{-}(5, x)$ & Bloch-Grünesen function & Eq. (28) (approximation) \\
\hline$v(y), t(y)$ & Fowler-Nordheim elliptical integral functions & $\ldots$ \\
\hline$\zeta(n)$ & Riemann zeta function & $\cdots$ \\
\hline
\end{tabular}

\section{FIELD ENHANCEMENT AND NOTIONAL EMISSION AREA}

A candidate to describe field enhancement for multiple protrusions is based on a point-charge model (PCM), the most familiar elementary example being a charge outside a flat metal surface, which generates a potential profile that can be modeled by the same charge and its image. For the PCM model of a cusp or bump, the model is to strategically place a point charge in the plane and consider the zero value equipotential line as the surface. A sequence of other point charges are then added to build up the cusp, such that along the symmetry axis, the first charge is placed at $a_{0}$, the second at $a_{1}$, and the $n$th at $a_{n-1}$. The impact on field 
enhancement, current density on axis, and total emission current from the bump so generated is then analytically tractable if it is assumed that $a_{n} / a_{n-1}=r$ for all $n$, where $r$ is a dimensionless factor relating a bump to the next larger bump. The heights of the bumps are defined by

$$
z_{n}=\sum_{j=0}^{n-1} a_{j}=\sum_{j=1}^{n} r^{j-1} a_{0}=\left(\frac{1-r^{n}}{1-r}\right) a_{0} \equiv S_{n}(r) a_{0}
$$

which defines the function $S_{n}(r)$. Significantly, if $r$ is small, then even though the bumps on the apex cannot be discerned visually on a length scale appropriate to the base, their impact on the field enhancement factor will nevertheless be pronounced (as shall be shown): it is worth emphasizing that this approach can easily treat protrusions whose dimensions would completely thwart a purely numerical (e.g., finite difference or finite element) approach. The potential is then defined by

$$
V_{n}(\rho, z)=F_{o} a_{0}\left\{-\frac{z}{a_{0}}+a_{0} \sum_{j=0}^{n} \frac{\lambda_{j}}{\sqrt{\rho^{2}+\left(z-z_{j}\right)^{2}}}\right\},
$$

where $F_{o}[\mathrm{eV} / \mathrm{nm}]$ is the background field, and the charge $\lambda_{n}$ of the $n$th point charge is fixed by demanding that the potential $V(\rho, z)$ satisfy $V\left(0, z_{n+1}\right) \equiv 0$ at the apex. An example for a larger $r$ of 0.75 is shown in Fig. 2 for the first few $n$; also shown is the $n=7$ and $r=0.25$ case (dashed line), showing the conelike tendency when $r$ is small. Note that the cylindrical coordinates $(\rho, z)$ scale with $a_{0}$. By specifying the value of $r$, structures between pyramids ( $r$ is small) and whiskers ( $r$ is near unity) can be generated. Though not used here, the formulation continues to hold for $r>1$.

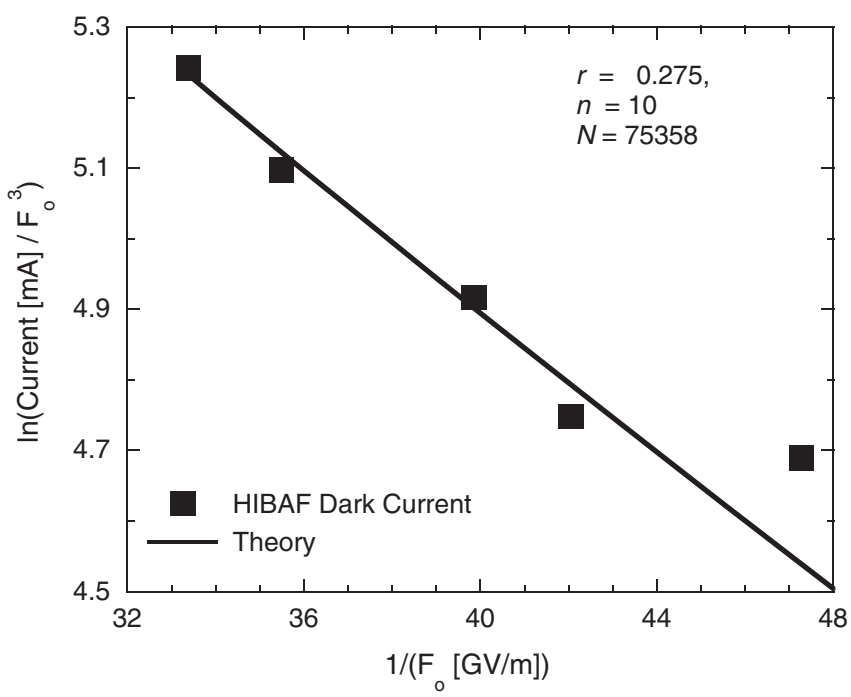

FIG. 6. Comparison of the data of Fig. 5 with the point-charge model coupled with a semiconductor field emission model to estimate of the factors $r, n$, and $N$ for the origins of field emission on the $\mathrm{CsK}_{2} \mathrm{Sb}$ photocathode surface in the Los Alamos high-brightness accelerator FEL (HIBAF).

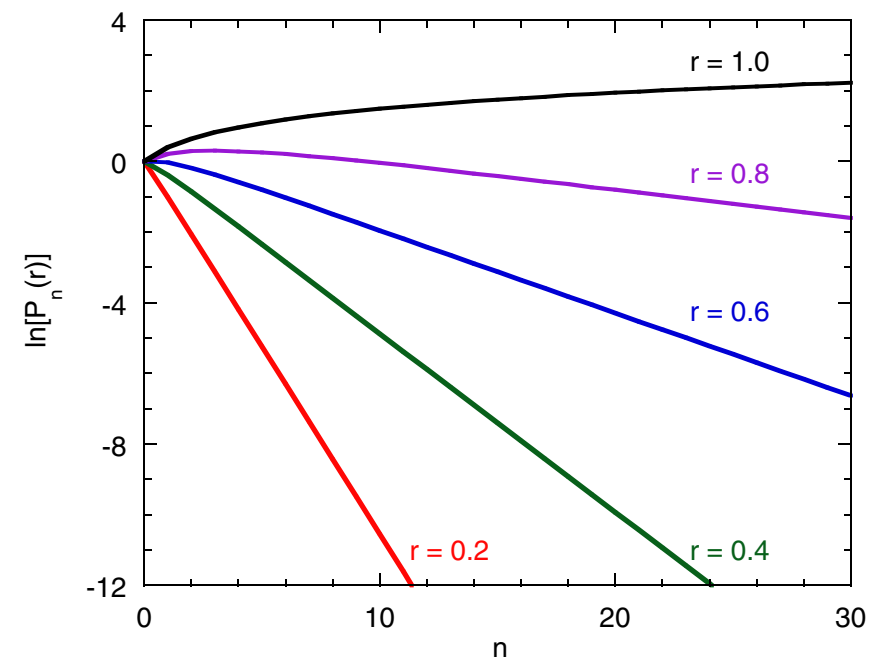

FIG. 7. (Color) The function $P_{n}(r)$ as a function of $n$ for various $r$.

The value of the $\lambda_{n}$ is ascertained from the boundary conditions (BC). Introducing the term $P_{n}(r) \equiv \lambda_{n} r^{-n}$ where $\lambda_{0}=1$, the $\mathrm{BC}$ give rise to the recursion relation

$$
P_{n}(r)=S_{n+1}(r)-\sum_{j=0}^{n-1} \frac{P_{j}(r)}{S_{n+1-j}(r)}
$$

It can be shown that $Q_{n}(r)=P_{n}(r) / r^{n}$ satisfies

$$
Q_{n}(r)=1+\sum_{j=0}^{n-1} \frac{Q_{j}(r)}{S_{n+1-j}(r) S_{n-j}(r)},
$$

from which it follows by $S_{n}(r=1) \approx 1$ and mathematical induction that in the limit of small $r, P_{n}(r=1) \approx 2^{n} r^{n}$. The behavior of $P_{n}(r)$ is shown in Fig. 7. The field enhancement factor $\beta_{n}$ and apex radius (i.e., radius of curvature for the equipotential line) $a_{\text {tip }}$ for the $n$th bump then can be shown to satisfy

$$
\begin{aligned}
\beta_{n}(r) & =1+a_{0}^{2} \sum_{j=0}^{n-1} \frac{\lambda_{j}}{\left(z_{n+1}-z_{j}\right)^{2}}=1+\sum_{j=0}^{n} \frac{P_{j}(r) r^{-j}}{\left[S_{n+1-j}(r)\right]^{2}} \\
a_{\text {tip }}(r) & =\left.\frac{\partial_{\rho} V}{\partial_{\rho}^{2} V}\right|_{\rho=0, z=z_{n+1}} \\
& =a_{0} \frac{\sum_{j=0}^{n} P_{j}(r) r^{2 n-j}\left[S_{n+1-j}(r)\right]^{-2}}{\sum_{j=0}^{n} P_{j}(r) r^{2(n-j)}\left[S_{n+1-j}(r)\right]^{-3}}
\end{aligned}
$$

where by field enhancement, it is meant the field at the apex by comparison to $F_{o}$. The behavior of the field enhancement factor for various bump numbers is shown in Fig. 8, along with approximations suggested by a multiplicative dependence $(r \ll 1$; red), a logarithmic dependence anticipated by a line charge model [54] $(r \approx 1$; purple), and an interpolation between these two limits (blue and green), or 


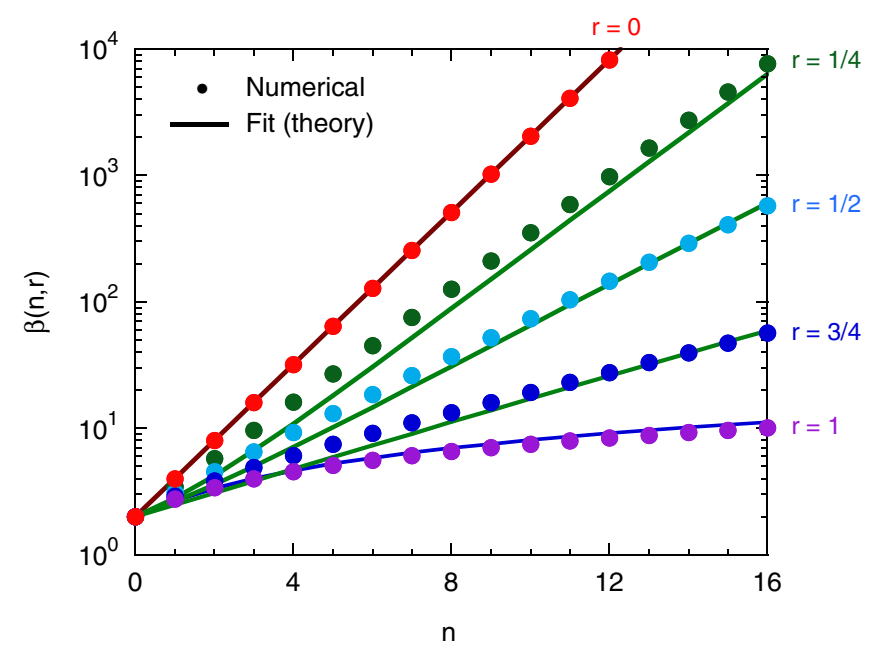

FIG. 8. (Color) The field enhancement factor $\beta_{n}(r)$ as a function of $n$ for various $r$. For small $r, \ln \left(\beta_{n}\right)$ is approximately linear, indicating that field enhancements are multiplicative.

$$
\beta_{n}(r) \approx \begin{cases}2^{n} & (r \rightarrow 0) \\ \frac{2(n+1) \ln (4)}{\ln [4(n+1)]} & (r \rightarrow 1) \\ 1+\frac{1}{2} \beta_{n}(0)\left[\frac{\beta_{n}(1)}{\beta_{n}(0)}\right]^{r} & (0<r<1)\end{cases}
$$

from which it is seen that, as with the findings of Miller et $a l$., the field enhancement factor is multiplicative only when $r$ is small.

The dependence of current on the rapidity with which $\beta_{n}(r)$ becomes large is, however, offset by the shrinkage of the apex radius, which, for small $r$, scales as $a_{n}(r=1) \approx$ $a_{0} r^{n}\left(2-2^{-n}\right)$, entailing that the notional emission area drops precipitously (in the case of Fig. 2 for the $r=0.25$ and $n=7$ apex, if $a_{o} \approx 100 \mu \mathrm{m}$, then $a_{7}=12 \mathrm{~nm}$ ). Care is therefore demanded in the evaluation of total current from the structure. Examples are shown in Figs. 9 and 10 for values of $r=0.25$ and 1.0, giving a sense of what "precipitous" entails: clearly, for small $r$, all but the merest fraction of the apex participates. Therefore, under small $r$ conditions, though field emission gives orders of magnitude larger current density in comparison to thermal emission, that is insufficient to categorically neglect the later: thermal emission will occur over a much larger region and can therefore give a nontrivial contribution to the bump current (an indication of this is shown in the Figs. 9 and 10 lines labeled FN and Richardson-LaueDushman (RLD), which are the field and thermal contributions as predicted using the conventional FN and RLD equations of the Appendices). A crude estimate using a hemispherical model [54] of the field variation shows that-using only the aforementioned conventional $\mathrm{FN}$ and RLD equations-for an apex field of $5 \mathrm{GV} / \mathrm{m}$, the FN and RLD total currents are roughly equal at a temperature of $2300 \mathrm{~K}$ for a work function of $4.6 \mathrm{eV}$.

Knowing the $V=0$ equipotential surface allows for the determination of the total current emitted from the struc-

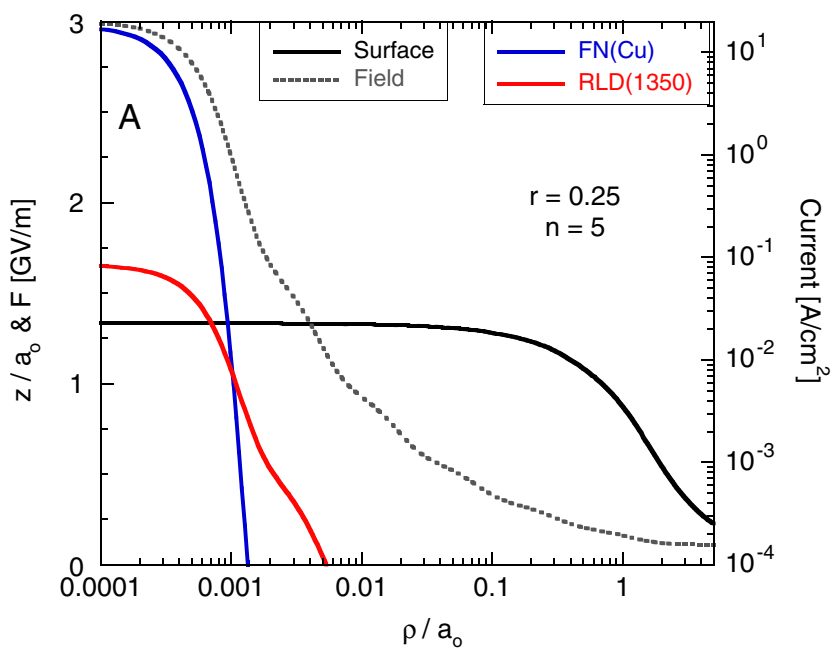

FIG. 9. (Color) The equipotential surface $z$ (black) and field (dashed) contrasted with the current density (blue for field, red for thermal) as a function of the cylindrical distance from the axis for small $r$ : note first that the field emission current dominates only very close to the apex, and second that both field and thermal current contributes.

ture. Analytical models of the notional emission area can be obtained in the same manner as for ellipsoidal and hyperbolic structures $[53,54,60]$; but in the present case, thermal contributions to the emitted current undercut that approach and we therefore resort to a numerical summation of the analytic terms. Therefore, the current from the structure is [61]

$$
\begin{aligned}
I_{\text {tip }} & =2 \pi \int_{0}^{L} \rho d \rho \sqrt{1+\left(\partial_{\rho} z\right)^{2}} J_{\mathrm{GTF}}[F(\rho), T] \\
& \approx \sum_{j=0}^{N-1}\left(2 \pi \Delta_{j}^{2}\right) S_{j} J_{\mathrm{GTF}}\left(F_{j}, T\right) N\left(\alpha_{j}\right),
\end{aligned}
$$

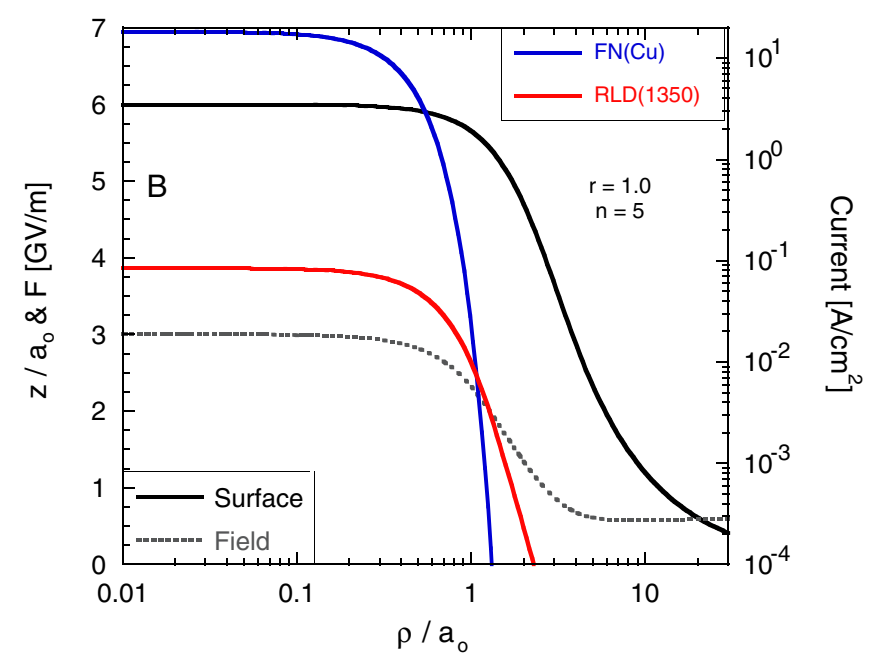

FIG. 10. (Color) Same as Fig. 9, but for $r=1$ for the same apex field. Field emission dominates over a much greater portion of the apex. 


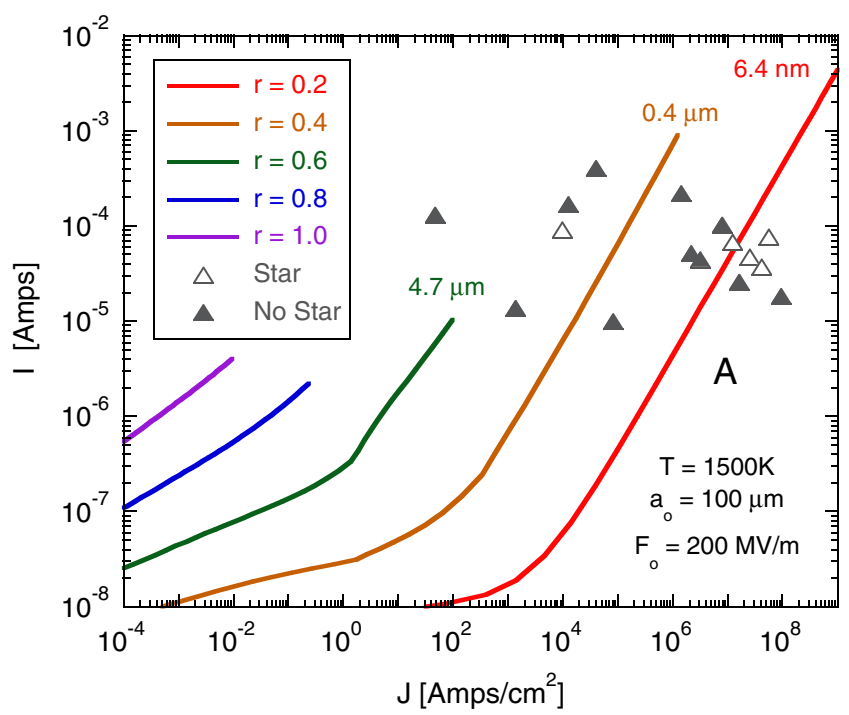

FIG. 11. (Color) Current versus current density for a given background field and temperature as the value of $n$ (number of point charges) increases. Numbers at the topmost end of the lines are the apex radii. "Star" and "No Star" are data points from Fig. 1 of Wilson (page 3 of Ref. [37]) for conditions leading to starburst formation and are provided for comparison. Increasing $n$ along such lines mimics asperity "growth."

where $\Delta_{j}^{2} \equiv\left(\rho_{j+1}^{2}-\rho_{j}^{2}\right) / 2, \alpha_{j} \approx \ln \left(J_{j} / J_{j+1}\right)$, the slope factor $S_{j}$ is the tilt of the $j$ th differential ribbon from which current is emitted-that is, $2 \pi \Delta_{j}^{2} S_{j}$ is the area of the differential ribbon - and the decay factor $N(\alpha)$ is due to the presumed variation of current as $J\left(\rho_{j} \leq \rho<\rho_{j+1}\right) \approx$ $J_{j} \exp \left[-\alpha_{j}\left(\rho-\rho_{j}\right) /\left(\rho_{j+1}-\rho_{j}\right)\right]$ or

$$
\begin{aligned}
S_{j} & \equiv \sqrt{1+\left(\frac{z_{j+1}-z_{j}}{\rho_{j+1}-\rho_{j}}\right)^{2}} ; \\
N(\alpha) & \equiv \frac{1}{\alpha^{2}}\left[1-(\alpha+1) e^{-\alpha}\right] .
\end{aligned}
$$

The total thermal-field emitted current $I_{\text {tip }}$ compared to the apex current density $J$ is shown for a variety of fields, temperatures, and $r$ factors in Figs. 11 and 12. As a comparison, the data showing the current and current density associated with "starburst" and "no-starburst" formations shown by Wilson [37] based on data by Knobloch is also shown - though it must be emphasized that multiple protrusions with a distribution of tip radii can be present in areas showing signs of explosive melting.

\section{NOTTINGHAM AND RESISTIVE HEATING}

As in the two data examples discussed previously, asperities on the surface that field emit demonstrate emission areas that are nanometer-scale in dimension, the bases of which are micron scale in turn. By comparison, the skin depth of most metals varies as $\delta[\mathrm{m}] \approx$ $503.3 \sqrt{\rho[\Omega-\mathrm{m}] / f[\mathrm{sec}]}$, where $\rho$ is the resistivity and $f$ is

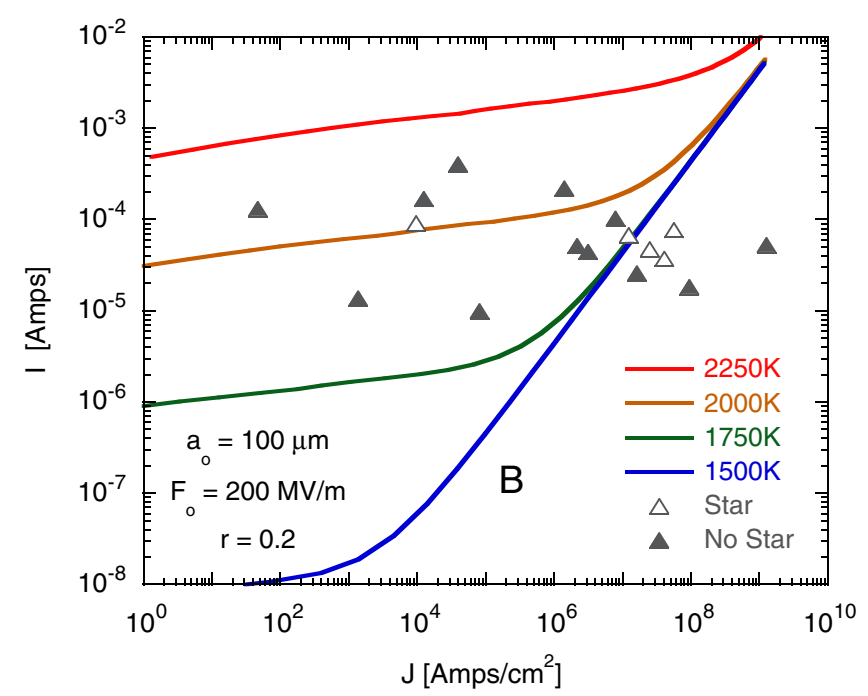

FIG. 12. (Color) Same as Fig. 11, but for fixed $n=6$ and different temperatures. Increasing $T$ along such lines models heating.

the modulation frequency. At rf frequencies, then, $\delta$ is of the same magnitude as the base of a presumed asperity: for example, at $750 \mathrm{MHz}$, the skin depths of $\mathrm{Al}, \mathrm{Cu}, \mathrm{Nb}, \mathrm{Mo}$, and $\mathrm{Mg}$ are 2.9, 2.3, 7.3, 4.0, and 3.7 microns, respectively. Therefore, to a first approximation, the current density over a cross section of an asperity-be it conical or whiskerlike-is uniform. An estimate of heating due to scattering (resistive heating) and electron emission (the Nottingham effect) may then be deduced from a one-dimensional model based on an approach suggested by Fursey [18]. The usage of 1D models gives estimates that are contingent, but the findings are reinforced by $3 \mathrm{D}$ numerical studies from the literature.

\section{A. The Nottingham effect}

Nottingham heating [62-64] and its impact on arcing and temperature rise in sharpened-and, in particular, microfabricated-structures has generated interest $[27,65,66]$ because of the suspected link between tip heating and failure. In particular, Miskovsky et al., argue that significant temperature rises occur near the apex when the cone angle is small (e.g., $15^{\circ}$ ) but further down the shank the temperature drops significantly, a finding supported by the work of Ancona as well as Fursey using 3D numerical approaches and treating heat diffusion. The source of "heating" arises when an electron is emitted below the Fermi level of the bulk metal: the electron within the metal that replaces the one lost is initially at the Fermi level, so that it gives up some energy to its surroundings. Conversely, electrons emitted above the Fermi level will tend to get replaced by electrons that must absorb energy, thereby leading to cooling. The average gain or loss of energy thereby gives rise to heating or cooling. The evalu- 
ation of the Nottingham effect proceeds from the definition of the average energy above or below the Fermi level of the emitted electrons as per

$$
\Delta E(F, T)=\langle E-\mu\rangle=\frac{\int(E-\mu) d J(E)}{\int d J(E)} .
$$

Clearly, the denominator of Eq. (11) gives $J_{\mathrm{GTF}}$, but where $E$ refers to the total energy of the electron, not [as in the formulation leading to Eq. (1) and as derived in the references] the energy component normal to the surface, or $E \cos ^{2} \theta$. That is, when $E$ is the total (not normal) energy, then

$$
J_{\mathrm{GTF}}(F, T)=\frac{2 A_{\mathrm{RLD}}}{k_{B}^{2}} \int_{0}^{\infty}\left[\int_{0}^{1} x T\left(E x^{2}\right) d x\right] f_{\mathrm{FD}}(E) E d E,
$$

where $T\left(E x^{2}\right)$ is the transmission probability, $x=\cos \theta$ is the angle with respect to normal at the surface, and $f_{\mathrm{FD}}(E)$ is the Fermi-Dirac distribution. The transmission probability is represented as $T\left(E x^{2}\right)=\left\{1+\exp \left[\beta_{F}\left(E_{o}-\right.\right.\right.$ $\left.\left.\left.E x^{2}\right)\right]\right\}^{-1}$, the angular integration is straightforward, and it can be shown that [after using $\exp \left(-E_{o} \beta_{F}\right) \ll 1$ ]

$$
\begin{aligned}
J_{\mathrm{GTF}}(F, T) & =\frac{A_{\mathrm{RLD}}}{k_{B}^{2}} \int_{0}^{\infty} \frac{\ln \left\{1+\exp \left[\beta_{F}\left(E-E_{o}\right)\right]\right\}}{1+\exp \left[\beta_{T}(E-\mu)\right]} d E \\
& \approx \frac{A_{\mathrm{RLD}}}{k_{B}^{2}}\left(\frac{n}{\beta_{T}}\right)^{2} \int_{-\infty}^{\infty} \frac{\ln \left(1+e^{-z}\right)}{1+e^{n(s-z)}} d z \\
& =\frac{A_{\mathrm{RLD}}}{k_{B}^{2} \beta_{T}^{2}} N(n, s),
\end{aligned}
$$

where in the first integral, $E$ refers to the total energy, the second line is the dimensionless integral form, and the third line can be shown by subjecting the integral in the second line to an integration by parts to obtain the form of $N(n, s)$ given as the large $u$ limit of Eq. [25] in Ref. [52] and given in Eq. (1). Greater accuracy is needed for $\Sigma(x)$.

\section{B. Approximations to $\Sigma(x)$}

Beginning with the definition of $\Sigma(x)$ in terms of the Riemann zeta function $\zeta(x)$ (a factor of 2 in the coefficient of the summation corrects the same formula in Refs. [44,52]),

$$
\Sigma(x)=1+2 \sum_{j=1}^{\infty}\left(1-2^{1-2 j}\right) \zeta(2 j) x^{2 j}
$$

the form of $\Sigma(x)$ can be written as the sum of singular term and a polynomial. The form of Eq. (14) appears to restrict the argument to less than unity, whereas in Eq. (1) the argument of one or the other of $\Sigma(n)$ or $\Sigma(1 / n)$ is larger than unity. The resolution is that $s$ tends to be large for emission conditions, and so either the first ("fielddominant") or second ("thermal-dominated") term in $N(n, s)$ survives for $n \neq 1$, though both are required for $n \approx 1$. As indicated before, a workable approach is to find an approximation to $\Sigma(x)$ valid for $x \leq 1$ that can be extended to $x>1$, as the exponentials of $e^{-s}$ and $e^{-n s}$ in Eq. (1) will quickly suppress the contribution of $\Sigma$ the argument greater than unity. Towards that objective, it can be shown that

$$
\begin{aligned}
\Sigma(x)= & 1+\frac{2 x^{2}\left(2+x^{2}\right)}{\left(1-x^{2}\right)\left(4-x^{2}\right)} \\
& +2 \sum_{j=1}^{\infty}\left(1-2^{1-2 j}\right)[\zeta(2 j)-1] x^{2 j} .
\end{aligned}
$$

The summation term is rapidly convergent. Though it appears enough to truncate the summation at the $j=2$ term, such an approximation would not satisfy (albeit by a small amount) the condition that $\Sigma(x)-\left[\left(1+x^{2}\right) /(1-\right.$ $\left.\left.x^{2}\right)\right] \rightarrow-1 / 2$ as $x$ goes to 1 , and so a term of order $x^{6}$ is added to enforce that condition. The final approximation becomes

$$
\begin{aligned}
\Sigma_{a}(x)= & \left(\frac{1+x^{2}}{1-x^{2}}\right)+\frac{1}{2} x^{2}\left(7 x^{4}-4 x^{2}-4\right)+\frac{1}{4} x^{2}\left(1-x^{2}\right) \\
& \times\left\{4 \zeta(2)+[7 \zeta(4)+4 \zeta(2)] x^{2}\right\} \\
= & \left(\frac{1+x^{2}}{1-x^{2}}\right)-0.039 x^{2}\left(9.1043+2.7163 x^{2}+x^{4}\right),
\end{aligned}
$$

where the " $a$ " subscript indicates that the expression is an approximation. Unless otherwise indicated, then, wherever $\Sigma(x)$ is required, Eq. (16) will be used. A related "derivative" function will also be required below: it is defined and approximated by

$$
\begin{aligned}
\Sigma^{\prime}(x) & \equiv x^{2} \frac{d}{d x}\left(\frac{\Sigma(x)}{x}\right) \\
\Sigma_{a}^{\prime}(x) & =\left(\frac{-1+4 x^{2}+x^{4}}{1-x^{2}}\right)+\frac{1}{4} x^{2}\left(-8-24 x^{2}+70 x^{4}-35 x^{6}\right)+\zeta(2) x^{2}\left(1-x^{4}+3 x^{6}\right)+\frac{7}{4} \zeta(4) x^{4}\left(3-5 x^{2}+2 x^{4}\right) \\
& =\left(\frac{-1+4 x^{2}+x^{4}}{1-x^{2}}\right)-0.027066 x^{2}\left(13.118+11.742 x^{2}+x^{6}\right)
\end{aligned}
$$




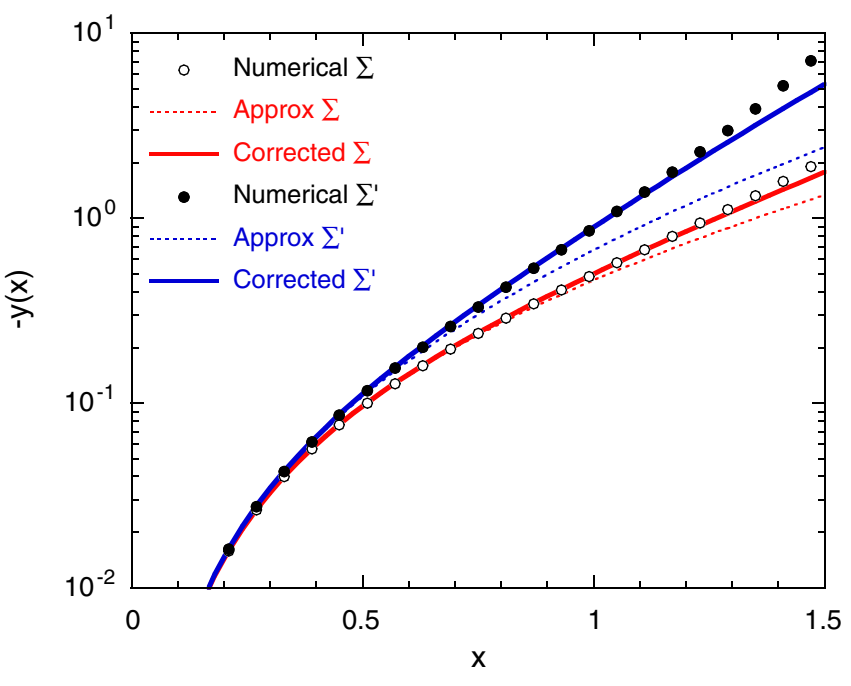

FIG. 13. (Color) Comparison of a numerical evaluation of $\Sigma(x)$ compared to truncating the series expansion of Eq. (15) at the second term (approximately $\Sigma$ ) and Eq. (16) (corrected $\Sigma$, or $\Sigma_{a}$ ): it is seen that $\Sigma_{a}$ performs very well through the $n \approx 1$ region.

where the correction term of order $x^{8}$ has been added to enforce the condition that $\Sigma^{\prime}(x)-\left[\left(x^{4}+4 x^{2}-1\right) /(1-\right.$ $\left.\left.x^{2}\right)^{2}\right] \rightarrow \zeta(2)-1$ as $x$ goes to 1 . The performance of the approximations are shown in Fig. 13, compared to the exact results [Eqs. (15) and (17)] as well as the approximations in which the summation in Eq. (15) is truncated at $j=2$, in which the $y$ axis is the difference between $\Sigma$ and its (singular at $x=1$ ) fraction term, and similarly for $\Sigma^{\prime}$.

\section{A Modified Nottingham $\Delta E$ term}

Using the same methodology that lead to the determination of $J_{\mathrm{GTF}}$ from its integral definition-namely, series expanding the components of the integrand fraction in Eq. (13) and integrating term by term using methods enumerated in Ref. [52] - a careful expansion of

$$
\begin{aligned}
& \int_{0}^{\infty}(E-\mu) \frac{\ln \left\{1+e^{\beta_{F}\left(E-E_{o}\right)}\right\}}{1+e^{\beta_{T}(E-\mu)}} d E \\
& =\frac{1}{\beta_{F}} \int_{0}^{\infty}(s-z) \frac{\ln \left\{1+e^{-z}\right\}}{1+e^{n(z-s)}} d z
\end{aligned}
$$

and the collection of leading order terms shows that $\Delta E$ in Eq. (11) is given by

$$
\Delta E=\frac{1}{n \beta_{F}}\left(\frac{N^{\prime}(n, s)}{N(n, s)}\right)
$$

where

$$
N^{\prime}(n, s) \equiv n^{3} \Sigma^{\prime}\left(\frac{1}{n}\right) e^{-n}+\left[(n s+1) \Sigma(n)-\Sigma^{\prime}(n)\right] e^{-n s},
$$

and where $n=\beta_{T} / \beta_{F}$ and $s=\beta_{F}\left(E_{o}-\mu\right)$. It is tempting to neglect terms proportional to $\exp (-n s)$, i.e., the

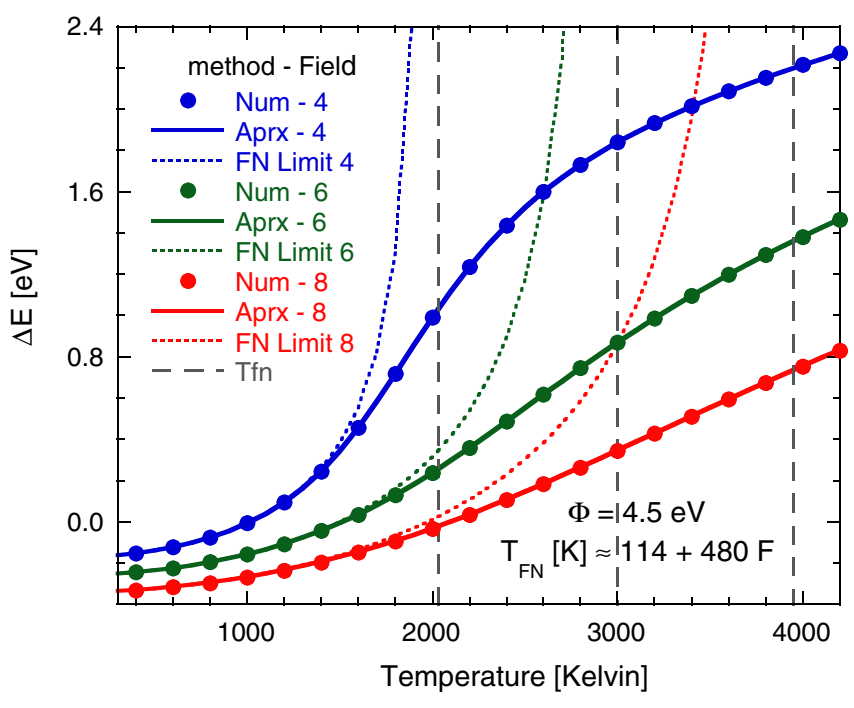

FIG. 14. (Color) The Nottingham term as a function of temperature for copperlike parameters calculated numerically (dots), using Eq. (19) (solid line), or using only the field-dominant terms of $N$ and $N^{\prime}$ containing $e^{-s}$ (the basis for the FN equation). The number in the legend corresponds to the apex field in $\mathrm{GV} / \mathrm{m} . T_{\mathrm{FN}}$ lines (vertical dashes) are where $\beta_{F}=\beta_{T}$.

thermal-dominated terms, and obtain the field-dominated limit $\Delta E \rightarrow \Sigma^{\prime}(1 / n) / \beta_{F} \Sigma(1 / n)$, but that would be in error. As emitter protrusions heat, they approach the transition regime where $n \approx 1$, indicated by when $T$ approaches $k_{B} T_{\mathrm{FN}}=1 / \beta_{F}(\mu)$ (referred to as $T_{\min }$ in Ref. [52]), and in that regime, the ratio of $\Sigma^{\prime}(1 / n)$ with $\Sigma(1 / n)$ is singular, whereas the ratio of $N^{\prime}(n, s)$ with $N(n, s)$ is not, with consequences as shown in Fig. 14. The pure numerical evaluation of Eq. (11) and the approximations using Eqs. (16), (17), and (19) compare very well, whereas the neglect of $e^{-n s}$ in $\Sigma(x)$ and its related term in $\Sigma^{\prime}(x)$ amounting to using only the pure field, or FN, limit-are increasingly inaccurate near the temperature where $n=1$ (shown by the vertical dashed lines and defined as $T_{\mathrm{FN}}$, below which field components dominate thermal components) and so reveal the limitations of using a pure FN approach to modeling Nottingham heating. Observe that for high fields (e.g., $8 \mathrm{GV} / \mathrm{m}$ ), Nottingham heating transitions to Nottingham cooling for temperatures higher than $2100 \mathrm{~K}$.

\section{Nottingham heating, resistive heating, and the heat diffusion equation}

The amount of heat deposited near the emission site per unit time is related to $\Delta E$ and the number of electrons emitted per second, or $I_{\text {tip }} / q$, where $q$ is the unit charge. If the radius of the emitter tip is $r_{e}$, then for a conical shape, the heating rate at the apex is approximately

$$
\Delta E\left(\frac{I_{\mathrm{tip}}}{q}\right) \approx \Delta E J_{\mathrm{GTF}} \Delta \Omega r_{e}^{2},
$$


where $\Delta \Omega$ is the solid angle of the cone. The amount of energy deposited in the cone apex volume $\Delta \Omega r_{e}^{3} / 3$ per unit time is then

$$
\Delta E\left(\frac{I_{\mathrm{tip}}}{q}\right)\left(\Delta \Omega \frac{1}{3} r_{e}^{3}\right)^{-1} \approx 3 \Delta E \frac{J_{\mathrm{GTF}}}{q r_{e}}
$$

which has units of power deposited per unit volume.

An approximation suggested by Fursey is to consider the apex of the emitter to be of a size comparable to the meanfree path of phonon scattering, so that the sphere of radius $r_{e}$ can be considered to be of a uniform temperature maintained by the Nottingham process: the approximation is more convenient than accurate here-for example, an emitter apex deforming under field and heating has a changing tip rather than a static one-but it is useful for determining the boundary condition for the heat diffusion equation. To that end, at the apex, the heat diffusion term $\vec{\nabla} \cdot(\kappa \vec{\nabla} T)$ integrated over the volume of the sphere is, in equilibrium, equal to the rate of energy deposited by Eq. (22). By Gauss' theorem, this amounts to

$$
\begin{aligned}
\int \vec{\nabla} \cdot(\kappa(T) \vec{\nabla} T) d V & =\kappa\left(T_{e}\right) \Delta \Omega r_{e}^{2}\left(\left.\partial_{r} T\right|_{r=r_{e}}\right) \\
& =\frac{3 \Delta E J_{\mathrm{GTF}}}{q r_{e}} \int_{0}^{r_{e}} r^{2} d r
\end{aligned}
$$

where $\kappa$ is the thermal conductivity, which identifies the boundary condition as

$$
\left.\partial_{r} T\right|_{r=r_{e}}=\frac{\Delta E J_{\mathrm{GTF}}}{q \kappa\left(T_{e}\right)} .
$$

Away from the apex, at a distance $r$, the current density is related to that at the apex by the constancy of total current, or $J(r)=J_{\mathrm{GTF}}\left(r_{e} / r\right)^{2}$. Resistive heating, which behaves as $\rho J^{2}$, therefore serves as a source term in the heat diffusion equation, which in radial coordinates and for equilibrium is now

$$
\kappa(T) \frac{1}{r^{2}} \partial_{r}\left(r^{2} \partial_{r} T\right)=-\rho(T) J_{\mathrm{GTF}}^{2}\left(\frac{r_{e}}{r}\right)^{4} .
$$

Integrating once, using the boundary conditions specified by Eq. (24), and integrating again gives, under the assumption that the variation in resistivity and thermal conductivity is weak,

$$
\begin{aligned}
T_{e}-T_{o}= & -\Delta E\left(\frac{T_{o}}{T}\right)\left(\frac{J_{\mathrm{GTF}}}{\kappa\left(T_{o}\right) q}\right)\left(\frac{r_{e}}{r_{o}}\right)\left(\frac{\rho(T)}{\rho\left(T_{o}\right)}\right)\left(r_{o}-r_{e}\right) \\
& +\frac{\rho\left(T_{o}\right)}{2 \kappa\left(T_{o}\right)}\left(\frac{T_{o}}{T}\right)\left[J_{\mathrm{GTF}}\left(\frac{r_{e}}{r_{o}}\right)\left(\frac{\rho(T)}{\rho\left(T_{o}\right)}\right)\left(r_{o}-r_{e}\right)\right]^{2},
\end{aligned}
$$

where the first term on the right-hand side is the Nottingham heating term, and the second is the resistive heating term. The subscript " $o$ " designates the base of the emitter, and " $e$ " conditions near the apex. Equation (26) is adequate to get a qualitative indication of the effects of current-induced temperature rise at the apex, but it is undercut by the presumed weakness of the temperature dependence of $\kappa$ and $\rho$. By virtue of its underlying approximations, particularly with regard to the temperature dependence of the resistivity and thermal conductivity, Eq. (26) bears differences to related expressions found by Fursey [Eq. (3.21) of Ref. [18]] and Charbonnier et al. [Eq. (9) of Ref. [55]].

The temperature dependence of the thermal conductivity and resistivity can be obtained by scaling arguments: both depend on the temperature-dependent relaxation time obtained from electron and phonon scattering [44]. Acoustic phonon scattering dominates, and so the resistivity can be expressed as

$$
\rho(T)=\rho\left(T_{o}\right)\left(\frac{T}{T_{o}}\right)^{5}\left\{\frac{W_{-}\left(5, T_{D} / T\right)}{W_{-}\left(5, T_{D} / T_{o}\right)}\right\}
$$

where $\rho\left(T_{o}\right)$ is the resistivity at a specific temperature such as room temperature (e.g., $152 \Omega$-nm and $15.4 \Omega$-nm for niobium and copper, respectively, for $T_{o}=300 \mathrm{~K}$ ), and $T_{D}$ is the Debye temperature (for a contrasting method of estimating the temperature dependence of resistivity as it relates to tip heating, see Ref. [23]). $W_{-}(n, x)$ is an integral related to Bose-Einstein statistics, and the special case for $n=5$ is known as the Bloch-Grüneisen function [67] for which a reasonable approximation is

$$
W_{-}(5, x) \approx \frac{120 \zeta(5) x^{5}}{1+\frac{80}{3} \zeta(5) x^{2}\left(1+18 x^{2}\right)} .
$$

From the relationship between resistivity and the relaxation time, it also follows that

$$
\kappa(T)=\kappa\left(T_{o}\right)\left(\frac{T}{T_{o}}\right)\left(\frac{\rho\left(T_{o}\right)}{\rho(T)}\right),
$$

where $\kappa\left(T_{o}\right)$ is the room-temperature value (e.g., $53.7 \mathrm{~W} / \mathrm{m}-\mathrm{K}$ and $401 \mathrm{~W} / \mathrm{m}-\mathrm{K}$ for niobium and copper, respectively). The Debye temperature to use is not without some ambiguity. The value of $275 \mathrm{~K}$ shall be used for niobium from Table I in Kittel [68]. Kittel also gives a value of $343 \mathrm{~K}$ for copper, but it is found using a leastsquares analysis that a value of $274.6 \mathrm{~K}$ causes the resistivity given by Eq. (27) for copper to lie directly upon the Bloch-Grüneisen line, and so the latter value shall be used here. In either case, the resistivity rapidly rises near the Debye temperature, but when the temperature is comparable to 5 times the Debye temperature, it levels off.

\section{ANALYSIS AND DISCUSSION}

The elements are now in place to find the temperature for which the right-hand side (RHS) of Eq. (26) is equal to the left (LHS) for a given field and base temperature under the assumption of the validity of the general thermal-field equation (the caveat is a reminder that the usage of the freeelectron formalism for metals such as tungsten is not 
without issues, as discussed by Charbonnier et al. [55]). Two separate circumstances are relevant. First, the base may be taken as a fixed value characteristic of the cathode surface in order to ascertain if Nottingham and resistive heating are significant for a given field. This case will be topical for considering whether emission heating sustains a large temperature that can contribute to surface migration and apex formation. Second, both the base and the apex may be thought of as having been brought to such a high temperature, regardless of how that is accomplished (e.g., back ion bombardment, emission heating, or both), that Nottingham cooling offsets resistive heating and the asperity is at a uniform temperature [both the LHS and RHS of Eq. (26) are zero]. This case will be topical for considering the limits on how hot the asperity may get if the heating mechanisms induce a run-away temperature growth; the expectation is that, at low fields, the level of heating will be controlled exclusively by the Nottingham effect, but at high fields, the resistive term can contribute.

Previous numerical simulations of the heating of microstructures has tended to use Fowler-Nordheim methodology $[69,70]$ or a numerical evaluation of the current density integrals [23,27], but extrapolations from them are nevertheless reasonable indications of trends. Very high temperatures at the apex of the emitter structure are entailed by high current and are predicted to reach, depending on circumstances, several thousand degrees (temperatures estimated by Charbonnier [25] or Fursey et al. [70] are suggested to be near $2000 \mathrm{~K}$ ) — that is, near levels required to melt some metals (e.g., copper has a melting point of $1358 \mathrm{~K}$ ) or provoke surface diffusion for otherwise rugged materials. Moreover, the temperature rise is, not surprisingly, found to be more pronounced the smaller the cone angle of the protrusions. The advantage of the point-charge method in relation to these numerical approaches is that far smaller features in relationship to the size of the presumed base of the asperity can be accounted for, emission from areas localized to but a few nanometers can thereby be effortlessly found, the relative magnitude of the competing effects can be discerned, and the temperature dependence of the important parameters are included.

For low temperatures, where the apex current density is not large, the majority of the heating is due to the Nottingham effect: a measure of how much is shown by the ratio of the resistive heating term with the Nottingham term, i.e., the ratio of the second term with the first term on the right-hand side of Eq. (26), which, under the approximation $r_{e}=r_{o}$, is given by

$$
y(F)=-\frac{q r_{e} \rho(T) J_{\mathrm{GTF}}(F, T)}{2 \Delta E(F, T)}
$$

and is shown in Fig. 15 for negative values of $\Delta E$. Such a comparison usefully reveals the dominance of Nottingham heating over resistive for generic conditions, but it is misleading in that the apex temperature cannot be taken as a

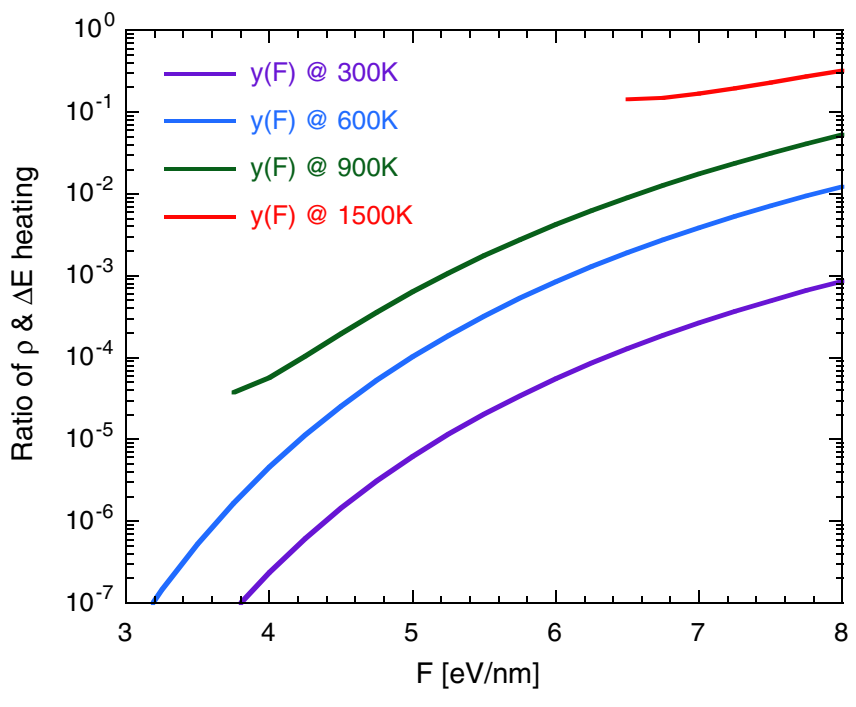

FIG. 15. (Color) Ratio of resistive heating to Nottingham heating (not cooling) for different temperatures as a function of apex field.

fixed value independent of field. Consider, then, the temperature of the apex when the base of an asperity is held at a fixed temperature. To anticipate temperature conditions that potentially had a hand in the creation of the asperity, it is assumed that the base is held at a fixed temperature of $T_{o}=600 \mathrm{~K}$ and is 10 microns in radius. The apex radius of the emitter is taken to be the same as the mean-free path of an electron, that is, the product of velocity at the Fermi level and the relaxation time, where the latter is inferred from the resistivity at room temperature [67] to be $43 \mathrm{~nm}$ for copperlike parameters. Holding the base at an artificially low temperature will force the apex temperature to

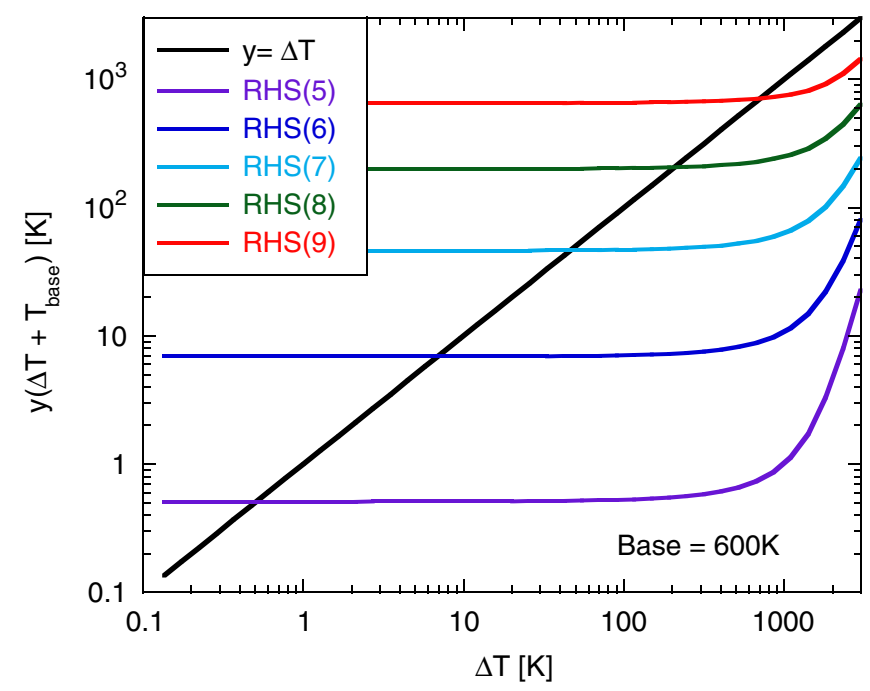

FIG. 16. (Color) Graphical representation of Eq. (26) as a function of temperature: the left-hand side is the solid black line. The right-hand side, or sum of the heating terms, are the solid colors. The base of the asperity is held at a fixed temperature of $600 \mathrm{~K}$. The number in parentheses in the legend refers to field in $\mathrm{GV} / \mathrm{m}$. 


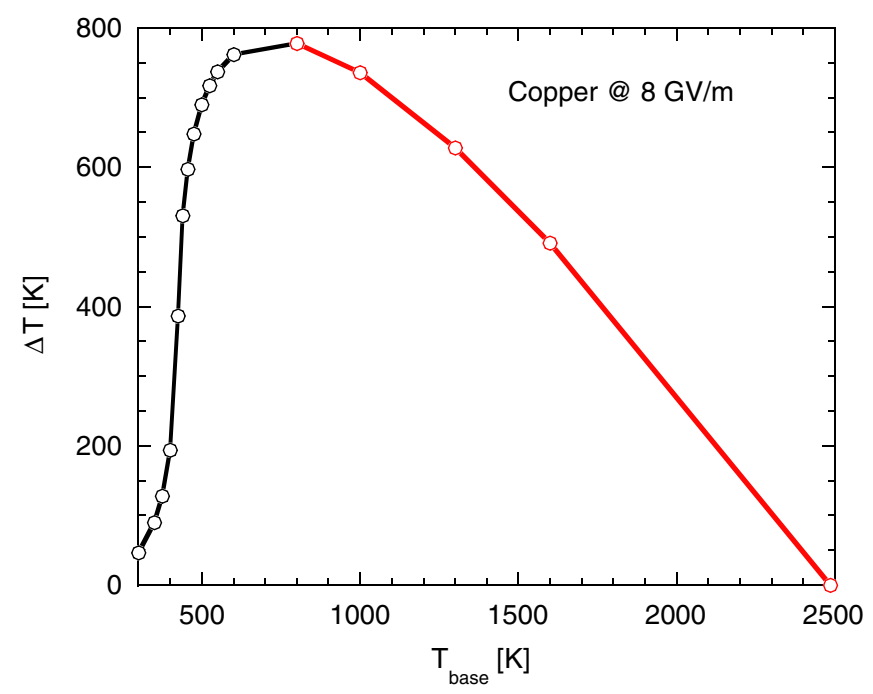

FIG. 17. (Color) Solutions to Eq. (26) for copperlike parameters, where $\Delta T$ is the left-hand side and $T_{\text {base }}=T_{o}$. As the base temperature increases, $\Delta T$ can increase markedly, even below the melting point.

be held low, but even so, significant temperature rises are anticipated. Solving Eq. (26) requires that the intersection of the LHS and the RHS be found as a function of apex temperature $T_{e}$ for a given base temperature $T_{o}$. An example is shown for the case $T_{o}=600 \mathrm{~K}$ in Fig. 16 for copperlike parameters: the intersection points of the diagonal with the horizontal lines are the equilibrium solutions for the apex temperature, and they are seen to progressively increase as the field increases. When the base temperature is high, it is expected that the curves will shift upwards, but that presents a difficulty in interpretation: the slope of the tangent line for the RHS at the intersection point approaches unity, indicating that the apex temperature can rise precipitously and thereby undercut the approximations leading to Eq. (26), or, in other words, the temperature rise can exhibit abrupt, or run-away, growth.

Instead of holding the base temperature at an arbitrarily fixed parameter, therefore, the field can be held constant and the question asked, for a given base temperature, what is the temperature rise at the apex for a given field? An example of such cumbersome and iterative calculations is shown in Fig. 17 for an assumed field of $8 \mathrm{eV} / \mathrm{nm}$ for copperlike parameters: the transition from black to red indicates those temperatures for which the apex temperature has exceeded the melting point of copper. It is seen that a rapid rise in the difference temperature is correlated with a rise in the base temperature, again suggesting that if a temperature rise occurred in the base, it could trigger a rapid temperature rise at the apex.

\section{SUMMARY}

A contributing factor to breakdown begins to emerge. As a consequence of local heating due to back ion bombardment, emission, or other mechanisms, material migration and tip formation serve to increase the field enhancement factor of an emerging protrusion produced by a continual increase in $n$ in Eq. (8) for a given $r$ in analogy with field forming of the apex during heating, as discussed by Schwoebel et al. [56]. Enhanced emission leads to greater heating of the apex; longer emission from the asperity leads to greater temperatures of the base of the asperity, and both lead to conditions in which the temperature can increase abruptly, either stressing the asperity to breakage or pushing its characteristic temperatures to the melting point. As has been shown, for $r=1 / 2$ or $1 / 4$, approximately seven bumps-on-bumps give rise to field enhancement factors of 26.2 or 75.5 , pushing macroscopic fields of order $100 \mathrm{MV} / \mathrm{m}$ into the field emission regime at the emission site rather easily.

Such a run-away scenario is not incompatible with failure mechanisms discussed by, for example, the melting or fracture models, heating and destruction models, and the creation and growth of nanoprotrusion models. What we have sought to show is that using (i) a correct expression for the thermal-field current, (ii) temperature-dependent resistivity and thermal conductivity values, (iii) models of field enhancement, and (iv) a one-dimensional radial heating diffusion model, a scenario by which a generated and heated protrusion experiences growth occurs that leads, in turn, to further heating and accelerated growth. These processes can then function in tandem with other macroscale mechanisms to lead to breakdown for parametric conditions that appear realizable.

\section{ACKNOWLEDGMENTS}

We thank the Joint Technology Office and the Office of Naval Research for supporting this work. We thank G. Nusinovich (UMD) for several beneficial discussions.

\section{APPENDIX A: THE FOWLER-NORDHEIM (FN) EQUATION}

The FN equation is an often used approximation in the modeling of field emission from microstructures [18,44,55,71-73] (see Ref. [74] for a review updating the equation itself and using contemporary values of the underlying physical constants). At the level of the Murphy and Good [51] formulation, it is represented as

$$
J_{\mathrm{FN}}(F)=a \frac{F^{2}}{\Phi t(y)^{2}} \exp \left(-\frac{b \Phi^{3 / 2}}{F} v(y)\right)
$$

where $F$ is the product of electron charge and electric field and therefore measured in $\mathrm{eV} / \mathrm{nm}$ (corresponding to an electric field of $\mathrm{GV} / \mathrm{m}$ ), and (compare [22], where the difference in units reflects the absorption of the charge $q$ into the field term $F$ ) 


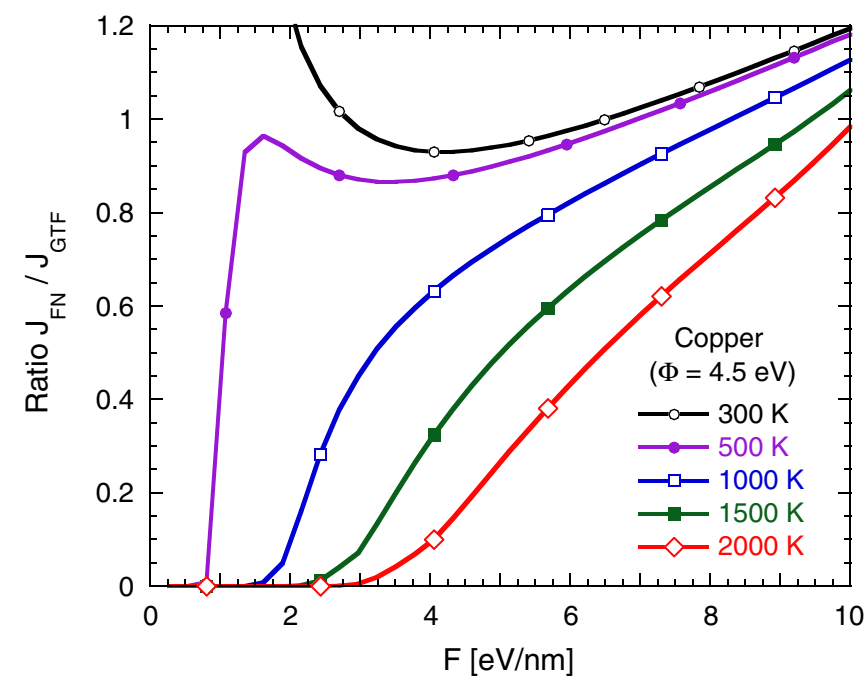

FIG. 18. (Color) Performance of the Fowler-Nordheim (FN) equation using the quadratic elliptical function approximations to the general thermal-field (GTF) equation. The FN equation in this approximation is temperature independent.

$$
\begin{aligned}
& a=\frac{q}{16 \pi^{2} \hbar}=1.5414 \times 10^{-6} \frac{\mathrm{Amp}}{\mathrm{eV}} \\
& b=\frac{4}{3 \hbar} \sqrt{2 m}=6.8309 \times 10^{9} \frac{1}{\sqrt{\mathrm{eV}} \text { meter }} \\
& y=\frac{\sqrt{\alpha_{f s} \hbar c F}}{\Phi}=3.7947 \times 10^{-5} \sqrt{\mathrm{eV}-\operatorname{meter}}\left(\frac{F^{1 / 2}}{\Phi}\right),
\end{aligned}
$$

where $\alpha_{f s}=1 / 137.036$ is the fine structure constant. Approximations are often invoked for the elliptical integral functions $t(y)$ and $v(y)$, the most common being to let the former be a constant and the later a quadratic function in $y$ : for example, Spindt et al. [71] choose $t(y)=\sqrt{1.1}$ and $v(y)=0.95-y^{2}$; Wang and Loew [38] choose $t(y)=1$ and $v(y)=0.956-1.062 y^{2}$; and finally, a requirement that the coefficient of $y^{2}$ be unity and using the Forbes approximation to the elliptical integrals [52,75] gives $t(y)=1.0613$ and $v(y)=0.93869-y^{2}$. It is the third representation that shall be used for comparison purposes here. A comparison of Eq. (A1) using Eq. (A2) to the general thermal-field equation given in Eq. (1) is shown in Fig. 18. In the cold or field emission limit, $\beta_{F}$ in Eq. (1) is evaluated at the Fermi level and is given by $\beta_{F}(\mu)=$ $(3 b / 2) \sqrt{\Phi} t(y) / F$ using the notation of Eq. (A2). The triangular barrier originally considered by Fowler and Nordheim corresponds to the limit $v(y) \approx t(y) \approx 1$.

\section{APPENDIX B: THE RICHARDSON-LAUE- DUSHMAN (RLD) EQUATION}

At the level of the Murphy and Good [51] formulation, the RLD equation is represented as

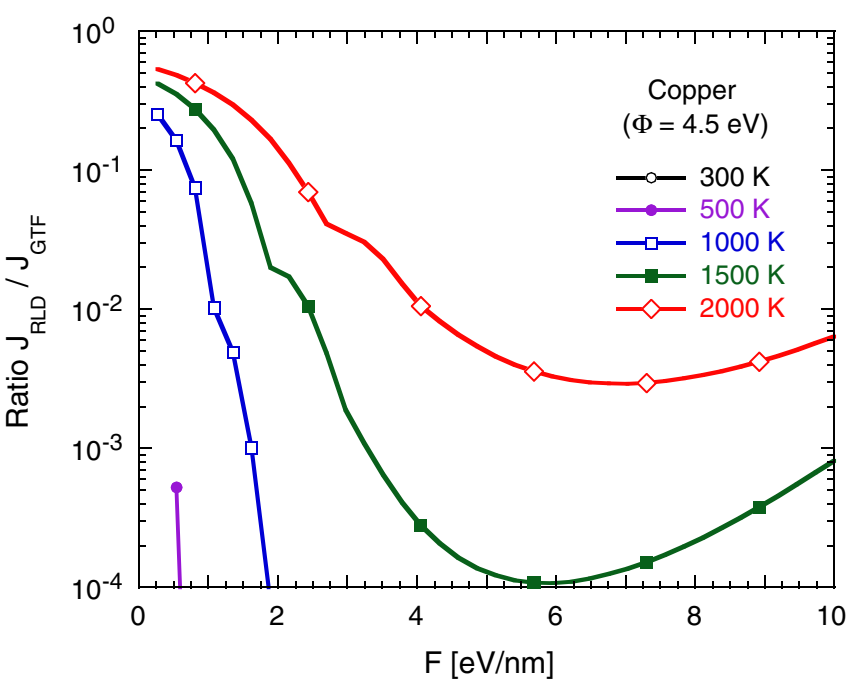

FIG. 19. (Color) Performance of the Richardson-Laue-Dushman (RLD) equation with Schottky barrier lowering to the general thermal-field (GTF) equation. The RLD equation in this approximation is field dependent through the factor $y$.

$$
J_{\mathrm{RLD}}(T, F)=a\left(\frac{3}{2} b\right)^{2} \frac{1}{\beta_{T}^{2}} \exp \left[-\beta_{T} \Phi(1-y)\right]
$$

in the notation of Eq. (A2), where $\beta_{T}=1 / k_{B} T$. The quantity $A_{\mathrm{RLD}}=a\left(3 k_{B} b / 2\right)^{2}=120.17 \mathrm{Amp} / \mathrm{K}^{2} \mathrm{~cm}^{2}$ is the Richardson constant. The factor $(1-y)$ in the exponential accounts for Schottky barrier lowering. In thermal emission limit, $\beta_{F}$ in Eq. (1) is evaluated at $\mu+\phi$, where $\phi=(1-y) \Phi$ for which $\beta_{F}(\mu+\phi)=(3 \pi b / 8) \times$ $\sqrt{2 y \Phi}(1-y) / F$ using the notation of Eq. (A2). A comparison of Eq. (B1) to the general thermal-field equation given in Eq. (1) is shown in Fig. 19.

[1] P. G. O'Shea, S. C. Bender, B. E. Carlsten, J. W. Early, D. W. Feldman, R. B. Feldman, W. J. D. Johnson, A. H. Lumpkin, R. I. Sheffield, R. W. Springer, W. E. Stein, and L. M. Young, Conference Record of the 1991 IEEE Particle Accelerator Conference 2754, 1991.

[2] A.H. Lumpkin, Conference Record of the 1991 IEEE Particle Accelerator Conference 1967, 1991.

[3] M. Jimenez, R. Noer, G. Jouve, C. Antoine, J. Jodet, and B. Bonin, J. Phys. D 26, 1503 (1993).

[4] J. Tan, H. Safa, B. Bonin, and M. Jimenez, J. Phys. D 27, 2654 (1994).

[5] M. Jimenez, R. Noer, G. Jouve, J. Jodet, and B. Bonin, J. Phys. D 27, 1038 (1994).

[6] B. Bonin, Vacuum 46, 907 (1995).

[7] S. Maïssa, T. Junquera, M. Fouaidy, A. Legoff, B. Bonin, M. Luong, H. Safa, and J. Tan, Appl. Surf. Sci. 94-95, 136 (1996).

[8] S. H. Gold and G. S. Nusinovich, Rev. Sci. Instrum. 68, 3945 (1997). 
[9] M. Gopych, H.-D. Graf, U. Laier, W. F. O. Muller, M. Platz, A. Richter, S. Setzer, A. Stascheck, S. Watzlawik, and T. Weiland, Nucl. Instrum. Methods Phys. Res., Sect. A 539, 490 (2005).

[10] D. H. Dowell, F. K. King, R. E. Kirby, J. F. Schmerge, and J. M. Smedley, Phys. Rev. ST Accel. Beams 9, 063502 (2006).

[11] W. P. Dyke, J. Trolan, E. Martin, and J. P. Barbour, Phys. Rev. 91, 1043 (1953).

[12] W. W. Dolan, W. P. Dyke, and J. K. Trolan, Phys. Rev. 91, 1054 (1953).

[13] G. N. Fursey, IEEE Trans. Electr. Insul. 20, 659 (1985).

[14] N.S. Xu and R. V. Latham, J. Phys. D 27, 2547 (1994).

[15] J. Norem, V. Wu, A. Moretti, M. Popovic, Z. Qian, L. Ducas, Y. Torun, and N. Solomey, Phys. Rev. ST Accel. Beams 6, 072001 (2003).

[16] J. Norem, Z. Insepov, and I. Konkashbaev, Nucl. Instrum. Methods Phys. Res., Sect. A 537, 510 (2005).

[17] C. K. Sinclair, Nucl. Instrum. Methods Phys. Res., Sect. A 557, 69 (2006).

[18] G. Fursey, Field Emission in Vacuum Microelectronics (Kluwer Academic/Plenum Publishers, New York, 2005).

[19] R. H. Fowler and L. Nordheim, Proc. R. Soc. A 119, 173 (1928).

[20] E. C. Landahl, F. V. Hartemann, G. P. Le Sage, W.E. White, H. A. Baldis, C. V. Bennett, J.P. Heritage, N. C. Luhmann, and C. H. Ho, IEEE Trans. Plasma Sci. 26, 814 (1998).

[21] H. Padamsee and J. Knobloch, AIP Conf. Proc. 474, 212 (1999).

[22] R. G. Forbes and K. L. Jensen, Ultramicroscopy 89, 17 (2001).

[23] Z. Yu, S. Deng, S. Wu, X. Zheng, J. Chen, and N. Xu, Ultramicroscopy 79, 125 (1999).

[24] K. L. Jensen, D. W. Feldman, and P. G. O'Shea, J. Vac. Sci. Technol. B 23, 621 (2005).

[25] F. Charbonnier, J. Vac. Sci. Technol. B 16, 880 (1998).

[26] W. P. Dyke, J. Trolan, W. Dolan, and G. Barnes, J. Appl. Phys. 24, 570 (1953).

[27] M. G. Ancona, J. Vac. Sci. Technol. B 13, 2206 (1995).

[28] P. B. Wilson, Report No. SLAC-pub-9953, 2003.

[29] P. B. Wilson, Report No. SLAC-tn-06-003, 2006.

[30] P. B. Wilson, Report No. SLAC-pub-12354, 2007.

[31] Z. Insepov, J. H. Norem, and A. Hassanein, Phys. Rev. ST Accel. Beams 7, 122001 (2004).

[32] A. S. Gilmour, Microwave Tubes (Artech House, Dedham, MA, 1986).

[33] D. Dorozhkina, V. Semenov, T. Olsson, D. Anderson, U. Jordan, J. Puech, L. Lapierre, and M. Lisak, Phys. Plasmas 13, 013506 (2006).

[34] D. C. Nguyen, P. L. Colestock, S. S. Kurennoy, D. E. Rees, A. H. Regan, S. Russell, D. L. Schrage, R. L. Wood, L. M. Young, T. Schultheiss, V. Christina, M. Cole, J. Rathke, J. Shaw, C. Eddy, R. Holm, R. Henry, and J. Yater, Nucl. Instrum. Methods Phys. Res., Sect. A 528, 71 (2004).

[35] B. Bonin, ICTP School on Nonaccelerator Particle Astrophysics, 1995.

[36] R. G. Forbes, Ultramicroscopy 89, 1 (2001).
[37] P. B. Wilson, High Gradient Workshop, 2007.

[38] J. W. Wang and G. A. Loew, Report No. SLAC-pub-7684, 1997.

[39] K. L. Jensen and C. Marrese-Reading, J. Vac. Sci. Technol. B 21, 412 (2003).

[40] M. Cahay, K. L. Jensen, and P. vonAllmen, in Noise and Fluctuations Control in Electronic Devices, edited by A. A. Balandin (American Scientific Publishers, Stevenson Ranch, CA, 2002).

[41] J. W. Wang, G. A. Loew, R. J. Loewen, R. D. Ruth, A. E. Vlieks, I. Wilson, and W. Wuensch, Proceedings of the Particle Accelerator Conference, Dallas, TX, 1995 (IEEE, New York, 1995), Vol. 1, p. 653.

[42] P. R. Schwoebel and C. A. Spindt, J. Vac. Sci. Technol. B 12, 2414 (1994).

[43] P. R. Schwoebel, C. A. Spindt, and C. Holland, J. Vac. Sci. Technol. B 21, 433 (2003).

[44] K. L. Jensen, Advances in Imaging and Electron Physics, Volume 149: Electron Emission Physics (Academic Press, New York, 2007).

[45] H. H. Braun, S. Döbert, I. Wilson, and W. Wuensch, Phys. Rev. Lett. 90, 224801 (2003).

[46] R. Stratton, Phys. Rev. 125, 67 (1962).

[47] A. Modinos, Surf. Sci. 42, 205 (1974).

[48] K. L. Jensen, B. L. Jensen, E. J. Montgomery, D. W. Feldman, P. G. O'Shea, and N. A. Moody (to be published).

[49] A.H. Sommer and W.E. Spicer, in Photoelectronic Materials and Devices, edited by S. Larach (Van Nostrand, Princeton, NJ, 1965), p. 175.

[50] P. Michelato, B. Di, A.C. Pagani, D. Sertore, and S. Valeri, Proceedings of the Particle Accelerator Conference, Dallas, TX, 1995 (IEEE, New York, 1995), Vol. 2, p. 1049.

[51] E. L. Murphy and R.H. Good, Phys. Rev. 102, 1464 (1956).

[52] K. L. Jensen, J. Appl. Phys. 102, 024911 (2007).

[53] K. L. Jensen, in Vacuum Microelectronics, edited by W. Zhu (Wiley, New York, 2001), p. 33.

[54] K. L. Jensen, J. Vac. Sci. Technol. B 21, 1528 (2003).

[55] F. M. Charbonnier, C. Bennette, and L. Swanson, J. Appl. Phys. 38, 627 (1967).

[56] P. R. Schwoebel, C. A. Spindt, C. Holland, and J. Panitz, J. Vac. Sci. Technol. B 19, 980 (2001).

[57] W. Schottky, Z. Phys. Hadrons Nucl. 14, 63 (1923).

[58] R. Miller, Y. Y. Lau, and J. H. Booske, Appl. Phys. Lett. 91, 074105 (2007).

[59] P. C. Bettler and F. M. Charbonnier, Phys. Rev. 119, 85 (1960).

[60] J. D. Zuber, K. L. Jensen, and T. E. Sullivan, J. Appl. Phys. 91, 9379 (2002).

[61] K. L. Jensen, D. W. Feldman, N. A. Moody, and P. G. O'Shea, J. Vac. Sci. Technol. B 24, 863 (2006).

[62] W. B. Nottingham, Phys. Rev. 49, 78 (1936).

[63] F. M. Charbonnier, R.W. Strayer, L.W. Swanson, and E. E. Martin, Phys. Rev. Lett. 13, 397 (1964).

[64] L. W. Swanson, L. C. Crouser, and F. M. Charbonnier, Phys. Rev. 151, 327 (1966).

[65] N. M. Miskovsky, S. H. Park, J. He, and P. H. Cutler, J. Vac. Sci. Technol. B 11, 366 (1993).

[66] J. Paulini, T. Klein, and G. Simon, J. Phys. D 26, 1310 
(1993).

[67] K. L. Jensen, N.A. Moody, D.W. Feldman, E. J. Montgomery, and P.G. O'Shea, J. Appl. Phys. 102, 074902 (2007).

[68] C. Kittel, Introduction to Solid State Physics (Wiley, New York, 1996).

[69] M. G. Ancona, Phys. Rev. B 46, 4874 (1992).

[70] G. N. Fursey, L. M. Baskin, D. V. Glazanov, A. N. Yevgen'ev, A. N. Kotcheryzhenkov, and S. A. Polezhaev, J. Vac. Sci. Technol. B 16, 232 (1998).
[71] C. A. Spindt, I. Brodie, L. Humphrey, and E. R. Westerberg, J. Appl. Phys. 47, 5248 (1976).

[72] P. R. Schwoebel and I. Brodie, J. Vac. Sci. Technol. B 13, 1391 (1995).

[73] A. Modinos, Field, Thermionic, and Secondary Electron Emission Spectroscopy (Plenum Press, New York, 1984).

[74] R. G. Forbes, Ultramicroscopy 79, 11 (1999).

[75] R. G. Forbes, Appl. Phys. Lett. 89, 113122 (2006). 